\title{
Ephemeroptera (Insecta) ocorrentes no Leste do Estado do Mato Grosso, Brasil
}

\author{
Yulie Shimano $^{1}$, Frederico Falcão Salles ${ }^{2}$ \& Helena Soares Ramos Cabette ${ }^{1}$ \\ ${ }^{1}$ Departamento de Ciências Biológicas, Universidade do Estado do Mato Grosso - UNEMAT, \\ CP 08, CEP 78690-000, Nova Xavantina, MT, Brasil \\ ${ }^{2}$ Centro Universitário Norte do Espírito Santo, \\ Universidade Federal de Espírito Santo - UFES, São Mateus, ES, Brasil \\ ${ }^{3}$ Autor para correspondência: Yulie Shimano,e-mail: yulie.bio@gmail.com
}

SHIMANO, Y., SALLES, F.F. \& CABETTE, H.S.R. Ephemeroptera (Insecta) from east of Mato Grosso State, Brazil. Biota Neotrop. 11(4): http://www.biotaneotropica.org.br/v11n4/en/abstract?inventory+bn02011042011

\begin{abstract}
Aiming to present a survey of Ephemeroptera fauna of Ephemeroptera from Mato Grosso State we investigated mayfly nymphs mainly from two hydrographic basins located in the eastern area of the state. Sixtyseven species/morphospecies in 41 genera and eight families were encountered. Four species (Cloeodes redactus Waltz \& McCafferty, 1987 and Waltzoyphius roberti Thomas \& Peru, 2002 [Baetidae], Tricorytopsis chiriguano Molineri, 2001 [Leptohyphidae] and Microphlebia surinamensis Savage \& Peters, 1983 [Leptophlebiidae]) are recorded for the first time from Brazil. The families Euthyplociidae, Polymitarcyidae and Coryphoridae are recorded for the first time from Mato Grosso, as well as 19 genera and 18 species. Twenty-five species could not be identified due to the lack of knowledge regarding the nymphal stage of their respective genera. As demonstrated in others surveys of mayflies in Brazil, the order is much more diverse than currently recognized, especially if we consider that several physiognomies and hydrographic basins of the state remain undocumented.
\end{abstract}

Keywords: Pindaíba River Basin, Suiá-Miçú River Basin, diversity, aquatic insects, nymphs.

SHIMANO, Y., SALLES, F.F. \& CABETTE, H.S.R. Ephemeroptera (Insecta) ocorrentes no Leste do Estado do Mato Grosso, Brasil. Biota Neotrop. 11(4): http://www.biotaneotropica.org.br/v11n4/pt/abstract?inventory +bn02011042011

Resumo: Com o objetivo de apresentar um levantamento da fauna de Ephemeroptera no Estado do Mato Grosso, investigamos as ninfas de Ephemeroptera, principalmente de duas bacias hidrográficas do leste do estado. Sessenta e sete espécies/morfoespécies em 41 gêneros e oito famílias foram encontradas. Quatro espécies (Cloeodes redactus Waltz \& McCafferty, 1987 e Waltzoyphius roberti Thomas \& Peru, 2002 [Baetidae], Tricorytopsis chiriguano Molineri, 2001 [Leptohyphidae] e Microphlebia surinamensis Savage \& Peters, 1983 [Leptophlebiidae]) foram registradas pela primeira vez no Brasil. As famílias Euthyplociidae, Polymitarcyidae e Coryphoridae foram registradas pela primeira vez para o Mato Grosso, assim como 19 gêneros e 18 espécies. Vinte e cinco espécies não foram identificadas devido à falta de informação envolvendo o estágio ninfal de seus respectivos gêneros. Assim como demonstrado em outros trabalhos com o grupo, a ordem se mostrou mais diversa do que se conhece atualmente, especialmente se considerarmos as várias fisionomias e bacias hidrográficas do estado que se mantêm desconhecidas.

Palavras-chave: Bacia do Rio Pindaíba, Bacia do Rio Suiá-Miçú, diversidade, insetos aquáticos, ninfas. 


\section{Introdução}

A ordem Ephemeroptera é um dos grupos de insetos aquáticos mais dominantes e diversos da América do Sul, e tem fascinado o homem por séculos devido a sua breve vida adulta comparada com o tempo de vida de suas ninfas (Domínguez et al. 2006). Esses organismos atingem sua maior diversidade em ambientes lóticos, onde compõem um importante elo na cadeia alimentar como consumidores primários de algas e perifíton e como alimento de consumidores secundários, como peixes e outros insetos (Brittain 1982, Salles 2006). Os efemerópteros estão entre os grupos mais utilizados em programas de biomonitoramento de qualidade de água em função das distintas respostas apresentadas por suas espécies à degradação ambiental (Domínguez et al. 2006, Salles 2006).

De acordo com a lista de espécies apresentada para o Brasil em 2004 (Salles et al. 2004a), que desde então é atualizada periodicamente na internet no sítio Ephemeroptera do Brasil (https:// sites.google.com/site/ephemeropterabr/), a ordem é constituída por 10 famílias, 68 gêneros e 211 espécies (última atualização em março de 2011). Porém, apesar do crescente número de trabalhos com registros e descrições de novas espécies para o Brasil (e.g. Dias et al. 2007a, 2008, 2009a,b, Salles \& Boldrini 2008, Salles \& Nieto 2008, Salles \& Polegatto 2008, Salles \& Nascimento 2009, Boldrini \& Salles 2009, Cruz et al. 2009, , Salles et al. 2009 , 2010b,c, Da-Silva et al. 2010 ,), acredita-se que o conhecimento a respeito da ordem no país ainda seja incipiente, uma vez que são poucos os trabalhos realizados a respeito do grupo em grande parte das regiões Nordeste e CentroOeste, e em muitas áreas da Região Norte.

No Estado de Mato Grosso são registradas cinco famílias (Baetidae, Caenidae, Leptohyphidae, Leptophlebiidae e Oligoneuriidae), 20 gêneros e 25 espécies de Ephemeroptera (Salles 2010), sendo esses os únicos registros para o Cerrado, o segundo maior bioma brasileiro em extensão, com 2 milhões de $\mathrm{km}^{2}$, aproximadamente $23 \%$ do território brasileiro (Ratter et al. 1997). Apesar de poucos, existe um número crescente de trabalhos realizados em diversos ambientes aquáticos no sudeste do estado, onde há novos registros e descrições de novos gêneros e espécies (e.g. Salles \& Batista 2004, Salles et al. 2004b, Polegatto \& Batista 2007, Dias et al. 2009a).

O Mato Grosso apresenta um mosaico de fisionomias (Pantanal, transição Floresta Amazônica-Cerrado e Cerrado) e uma extensa rede hidrográfica, o que pode acarretar numa ampla diversidade de insetos aquáticos e, consequentemente, de espécies de Ephemeroptera. Por outro lado, é um dos estados campeões em desmatamento e queimadas, sendo um dos principais responsáveis pelo avanço do desflorestamento do Bioma Amazônico (Schwartzman \& Zimmerman 2005). Sendo um grupo de insetos tão importante em monitoramento de qualidade de água e partindo da premissa que o desmatamento ocasiona a perda de diversidade, existe uma necessidade imediata de conhecimento de sua fauna.

É importante se conhecer a diversidade de espécies para a compreensão da natureza, assim como para gerenciá-la em relação às atividades de exploração, conservação de recursos naturais e recuperação de ecossistemas degradados (Melo 2008). Sendo assim, o objetivo deste trabalho é a apresentação de um inventário da fauna de Ephemeroptera para o Estado de Mato Grosso, abrangendo a sua região leste, a qual engloba áreas de Cerrado e de transição CerradoAmazônia, incluindo discussões acerca de aspectos da biologia dos táxons no estágio ninfal, o qual foi utilizado para o presente levantamento.

\section{Material e Métodos}

\section{1. Área de estudo}

Os dados desse inventário se basearam principalmente em duas bacias hidrográficas situadas na porção leste do Estado do Mato
Grosso (Figura 1), sendo a Bacia do Rio Pindaíba situada em áreas de predomínio de Cerrado e a Bacia do Rio Suiá-Miçú em áreas de transição entre a Floresta Amazônica e o Cerrado, coletados entre os anos de 2005 e 2008. Também foram acrescentadas informações de coletas avulsas realizadas nos anos 1999 a 2005 em outros córregos do Mato Grosso, fora das bacias citadas (Tabela 1). Dados de literatura também foram usados a fim de complementar o presente trabalho.

A Bacia Hidrográfica do Rio Pindaíba faz parte da Bacia Hidrográfica do Rio das Mortes e abrange parte dos municípios de Barra do Garças, Araguaiana, Cocalinho e Nova Xavantina, situando-se mais ao sul do estado. O Rio Pindaíba deságua no Rio das Mortes, com a maior parte das nascentes inseridas nas terras altas, com altitudes médias de $600 \mathrm{~m}$, que compõem os Planaltos dos Guimarães, vindo a desenvolver seu curso nas áreas da planície, com altitudes médias de $330 \mathrm{~m}$. As principais atividades econômicas desenvolvidas na Bacia são a pecuária de corte e, secundariamente, a agricultura (Rossete 2005). O clima predominante na região é do tipo tropical, com duas estações bem definidas: um período de seca, de maio até setembro e um período chuvoso de novembro a março (Brasil 1981, Vianello \& Alves 2000).

A Bacia do Rio Suiá-Miçú possui mais de 2 milhões de ha e é um dos principais afluentes do Rio Xingu, e está localizada nos municípios de Querência, Ribeirão Cascalheira e Canarana, situada mais ao norte de Mato Grosso. A Bacia do Rio Suiá-Miçú abriga trechos ainda preservados de tipos vegetacionais distintos onde, segundo Ratter et al. (1978), merecem destaque as fisionomias de cerrado sensu stricto, cerrado transicional denominado cerradão de vermelhão (Hirtella glandulosa Spreng.-Chrysobalanaceae) e áreas de Mata Seca. Os tributários formadores do Rio Suiá-Miçú apresentam distinção nas suas características relacionadas ao tipo de formação geológica e de vegetação ripária, com mosaicos de nascentes ritrais encaixadas de maior declividade, mata de galeria típica e nascentes alagadas com presença de macrófitas, buritizais e maior entrada de luz. As principais atividades econômicas desenvolvidas nesta área são a extração de madeira, monoculturas de arroz e soja e pecuária extensiva (Riva et al. 2007). De acordo com Ratter et al. (1978), a região apresenta clima tropical sazonal com estação seca de maio a outubro e chuvosa de novembro a abril, sendo do Subtipo Savana $(A w)$ e com microrregiões do Subtipo Monções $(A m)$ e Tropical Chuvoso $(A)$ segundo classificação de Köppen, com precipitação média de $1.370 \mathrm{~mm}$ e temperatura entre $32,7 \mathrm{e} 17,0^{\circ} \mathrm{C}$.

Em ambas as bacias foram realizadas coletas em três estações. $\mathrm{Na}$ Bacia do Rio Pindaíba as coletas ocorreram no auge da chuva (janeiro/2005), na seca (julho e agosto/2005) e no início das chuvas (outubro e novembro/2005), enquanto que na Bacia do Rio SuiáMiçú houve coletas na seca (setembro/2007), início das chuvas (dezembro/2007) e início da seca (maio/2008).

\section{Procedimentos de coleta e identificação}

Ao todo foram amostrados 42 córregos, sendo que em 35 deles, a metodologia empregada consistiu em coletas quantitativas das ninfas de Ephemeroptera, obtidas através de um coador de $18 \mathrm{~cm}$ de diâmetro e malha de $0,05 \mathrm{~mm}$ (rapiché) em transecções de $100 \mathrm{~m}$. Nos demais córregos foram realizadas coletas avulsas através de draga ou rede surber. O material foi separado em campo e conservado em álcool etílico a $85 \%$.

Em laboratório, os espécimes foram identificados com o auxílio de chaves de identificação de Salles (2006), Domínguez et al. (2006), Dias et al. (2007b), artigos e descrições de espécies, todos disponibilizados no sítio Ephemeroptera Galactica (http://www.famu. org/mayfly/). O material foi depositado na Coleção Zoobotânica James Alexander Ratter, no campus de Nova Xavantina, Universidade do Estado do Mato Grosso - UNEMAT. 

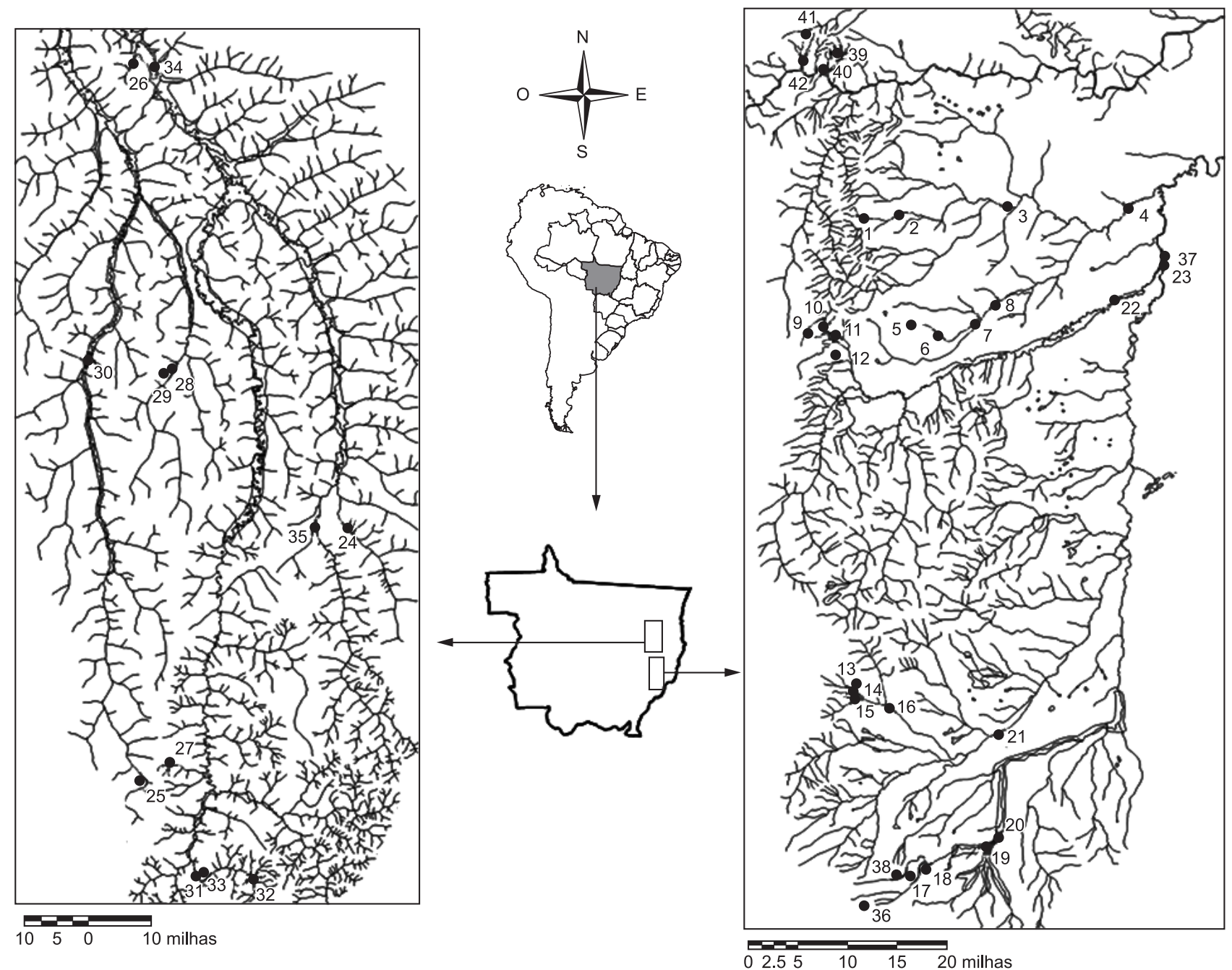

Figura 1. Pontos de coleta de Ephemeroptera imaturos nas Bacias do Rio Suiá-Miçú (à esquerda) e Rio Pindaíba e Mortes (à direita), Mato Grosso (números correspondem aos locais, ver Tabela 1).

Figure 1. Samples sites of immature Ephemeroptera from Suiá-Miçú (left) and Pindaíba and Mortes (right) Rivers Basins, Mato Grosso (numbers correspond to localities, see Table 1).

\section{Distribuição geográfica e material examinado}

Foram consultadas informações de Salles (2010) e artigos de Nolte et al. (1997), Lima et al. (2010) e Salles et al. (2010b,c). As siglas utilizadas seguem o padrão utilizado para a abreviação dos estados brasileiros.

Para os táxons com novos registros para o Mato Grosso e espécies que não foram identificadas, foram atribuídos alguns comentários. No material examinado apresentamos os dados da seguinte forma: PT número (ponto de coleta, para maiores informações ver Tabela 1), seguido do número de indivíduos entre parênteses e, por fim, a data da coleta.

\section{Resultados e Discussão}

\section{Baetidae}

A família Baetidae, descrita por Leach em 1815, é amplamente distribuída, podendo ser encontrada em todos os continentes e em várias ilhas (Edmunds Junior et al. 1976), e é uma das mais diversas dentro da ordem, perdendo apenas para Leptophlebiidae (Domínguez et al. 2006).

Seus representantes habitam uma variedade de ambientes, de lóticos a lênticos em substratos arenosos ou compostos por pedra (Domínguez et al. 2006, Salles 2006). Estudos e observações realizadas por Buss \& Salles (2007) relataram variação quanto às exigências das espécies de Baetidae por locais com integridade ambiental mais elevada e tipos de substratos associados.

No Mato Grosso, estudos realizados em diversos ambientes aquáticos do município de Nova Xavantina e arredores, sudeste do Mato Grosso, vêm demonstrando que a fauna de Baetidae da região é bem diversificada (Salles et al. 2004b).

Americabaetis alphus Lugo-Ortiz \& McCafferty, 1996

Distribuição: AL, AM, BA, ES, GO, MG, MT, PR, RJ, RS, SE, SC e SP

Aspectos biológicos: Coletados em córregos de $1^{\mathrm{a}}$ a $5^{\mathrm{a}}$ ordem da Bacia do Rio Pindaíba em trechos conservados e alterados, alguns indivíduos estavam associados ao substrato madeira. Na Bacia do 
Shimano, Y. et al

Tabela 1. Pontos de coletas em rios e córregos no leste do Estado de Mato Grosso, local, bacia e coordenadas geográficas, 1999/2008.

Table 1. Samples sites in Rivers and streams from West of Mato Grosso State, local, Basin and geographic coordinates, 1999/2008.

\begin{tabular}{|c|c|c|c|}
\hline Ponto & Local & Bacia & Coordenadas \\
\hline PT 01 & Córrego Cachoeirinha- ${ }^{a}$ ordem & $\mathrm{RP}$ & $14^{\circ} 50^{\prime} 30^{\prime \prime} \mathrm{S}$ e $52^{\circ} 24^{\prime} 54^{\prime \prime} \mathrm{O}$ \\
\hline PT 02 & Córrego Cachoeirinha- $2^{\mathrm{a}}$ ordem & $\mathrm{RP}$ & $14^{\circ} 50^{\prime} 50^{\prime \prime} \mathrm{S}$ e $52^{\circ} 24^{\prime} 22^{\prime \prime} \mathrm{O}$ \\
\hline PT 03 & Córrego Cachoeirinha- $3^{\mathrm{a}}$ ordem & $\mathrm{RP}$ & $14^{\circ} 50^{\prime} 33^{\prime \prime} \mathrm{S}$ e $52^{\circ} 21^{\prime} 34^{\prime \prime} \mathrm{O}$ \\
\hline PT 04 & Córrego Cachoeirinha- $4^{\mathrm{a}}$ ordem & $\mathrm{RP}$ & $14^{\circ} 49^{\prime} 45^{\prime \prime} \mathrm{S}$ e $52^{\circ} 12^{\prime} 55^{\prime \prime} \mathrm{O}$ \\
\hline PT 05 & Córrego Caveira- $1^{\mathrm{a}}$ ordem & $\mathrm{RP}$ & $14^{\circ} 59^{\prime} 06^{\prime \prime} \mathrm{S}$ e $52^{\circ} 20^{\prime} 29^{\prime \prime} \mathrm{O}$ \\
\hline PT 06 & Córrego Caveira- $2^{\mathrm{a}}$ ordem & $\mathrm{RP}$ & $14^{\circ} 59^{\prime} 53^{\prime \prime} \mathrm{S}$ e $52^{\circ} 18^{\prime} 17^{\prime \prime} \mathrm{O}$ \\
\hline PT 07 & Córrego Caveira- $3^{\mathrm{a}}$ ordem & $\mathrm{RP}$ & $14^{\circ} 57^{\prime} 28^{\prime \prime} \mathrm{S}$ e $52^{\circ} 13^{\prime} 43^{\prime \prime} \mathrm{O}$ \\
\hline PT 08 & Córrego Caveira- $4^{\mathrm{a}}$ ordem & $\mathrm{RP}$ & $14^{\circ} 49^{\prime} 47^{\prime \prime} \mathrm{S}$ e $52^{\circ} 03^{\prime} 16^{\prime \prime} \mathrm{O}$ \\
\hline PT 09 & Córrego da Mata-1 ordem & $\mathrm{RP}$ & $14^{\circ} 59^{\prime} 53^{\prime \prime} \mathrm{S}$ e $52^{\circ} 28^{\prime} 42^{\prime \prime} \mathrm{O}$ \\
\hline PT 10 & Córrego da Mata-2 ordem & $\mathrm{RP}$ & $14^{\circ} 59^{\prime} 18^{\prime \prime} \mathrm{S}$ e $52^{\circ} 27^{\prime} 30^{\prime \prime} \mathrm{O}$ \\
\hline PT 11 & Córrego da Mata-3 ordem & $\mathrm{RP}$ & $14^{\circ} 59^{\prime} 59^{\prime \prime} \mathrm{S}$ e $52^{\circ} 26^{\prime} 29^{\prime \prime} \mathrm{O}$ \\
\hline PT 12 & Córrego da Mata-4 ordem & $\mathrm{RP}$ & $15^{\circ} 01^{\prime} 32^{\prime \prime} \mathrm{S}$ e $52^{\circ} 26^{\prime} 29^{\prime \prime} \mathrm{O}$ \\
\hline PT 13 & Córrego Papagaio- $1^{\mathrm{a}}$ ordem & $\mathrm{RP}$ & $15^{\circ} 27^{\prime} 01^{\prime \prime} \mathrm{S}$ e $52^{\circ} 24^{\prime} 30^{\prime \prime} \mathrm{O}$ \\
\hline PT 14 & Córrego Papagaio- $2^{\mathrm{a}}$ ordem & $\mathrm{RP}$ & $15^{\circ} 27^{\prime} 32^{\prime \prime} \mathrm{S}$ e $52^{\circ} 24^{\prime} 42^{\prime \prime} \mathrm{O}$ \\
\hline PT 15 & Córrego Papagaio- $3^{\mathrm{a}}$ ordem & $\mathrm{RP}$ & $15^{\circ} 28^{\prime} 11^{\prime \prime} \mathrm{S}$ e $52^{\circ} 24^{\prime} 32^{\prime \prime} \mathrm{O}$ \\
\hline PT 16 & Córrego Papagaio-4 ${ }^{\mathrm{a}}$ ordem & $\mathrm{RP}$ & $15^{\circ} 28^{\prime} 56^{\prime \prime} \mathrm{S}$ e $52^{\circ} 21^{\prime} 47^{\prime \prime} \mathrm{O}$ \\
\hline PT 17 & Córrego Taquaral- $1^{\mathrm{a}}$ ordem & $\mathrm{RP}$ & $15^{\circ} 41^{\prime} 54^{\prime \prime} \mathrm{S}$ e $52^{\circ} 20^{\prime} 03^{\prime \prime} \mathrm{O}$ \\
\hline PT 18 & Córrego Taquaral- $2^{\mathrm{a}}$ ordem & $\mathrm{RP}$ & $15^{\circ} 41^{\prime} 57^{\prime \prime} \mathrm{S}$ e $52^{\circ} 19^{\prime} 56^{\prime \prime} \mathrm{O}$ \\
\hline PT 19 & Córrego Taquaral- $3^{\mathrm{a}}$ ordem & $\mathrm{RP}$ & $15^{\circ} 39^{\prime} 35^{\prime \prime} \mathrm{S}$ e $52^{\circ} 13^{\prime} 52^{\prime \prime} \mathrm{O}$ \\
\hline PT 20 & Córrego Taquaral- $4^{\mathrm{a}}$ ordem & $\mathrm{RP}$ & $15^{\circ} 38^{\prime} 53^{\prime \prime} \mathrm{S}$ e $52^{\circ} 12^{\prime} 53^{\prime \prime} \mathrm{O}$ \\
\hline PT 21 & Rio Corrente- $5^{\mathrm{a}}$ ordem & $\mathrm{RP}$ & $15^{\circ} 31^{\prime} 14^{\prime \prime} \mathrm{S}$ e $52^{\circ} 12^{\prime} 10^{\prime \prime} \mathrm{O}$ \\
\hline PT 22 & Rio Pindaíba- $5^{\mathrm{a}}$ ordem & $\mathrm{RP}$ & $14^{\circ} 56^{\prime} 56^{\prime \prime} \mathrm{S}$ e $52^{\circ} 04^{\prime} 17^{\prime \prime} \mathrm{O}$ \\
\hline PT 23 & Rio Pindaíba- $6^{\mathrm{a}}$ ordem & $\mathrm{RP}$ & $14^{\circ} 54^{\prime} 10^{\prime \prime} \mathrm{S}$ e $52^{\circ} 00^{\prime} 21^{\prime \prime} \mathrm{O}$ \\
\hline PT 24 & Córrego Brejão & SM & $12^{\circ} 38^{\prime} 32,3^{\prime \prime} \mathrm{S}$ e $51^{\circ} 53^{\prime} 20,6^{\prime \prime} \mathrm{O}$ \\
\hline PT 25 & Córrego Lúcio & SM & $13^{\circ} 05^{\prime} 34,5^{\prime \prime} \mathrm{S}$ e $52^{\circ} 15^{\prime} 16,9^{\prime \prime} \mathrm{O}$ \\
\hline PT 26 & Córrego Sucuri & SM & $11^{\circ} 49^{\prime} 50,7^{\prime \prime} \mathrm{S}$ e $52^{\circ} 17^{\prime} 02,2^{\prime \prime} \mathrm{O}$ \\
\hline PT 27 & Córrego Transição Brejo & SM & $13^{\circ} 03^{\prime} 35,6^{\prime \prime} \mathrm{S}$ e $52^{\circ} 12^{\prime} 03,3^{\prime \prime} \mathrm{O}$ \\
\hline PT 28 & Rio Betis ponto 1 & SM & $12^{\circ} 22^{\prime} 28,7^{\prime \prime} \mathrm{S}$ e $52^{\circ} 13^{\prime} 23,1^{\prime \prime} \mathrm{O}$ \\
\hline PT 29 & Rio Betis ponto 2 & SM & $12^{\circ} 22^{\prime} 27,5^{\prime \prime} \mathrm{S}$ e $52^{\circ} 13^{\prime} 19,0^{\prime \prime} \mathrm{O}$ \\
\hline PT 30 & Rio Darro & SM & $12^{\circ} 21^{\prime} 12,3^{\prime \prime} \mathrm{S}$ e $52^{\circ} 21^{\prime} 27,4^{\prime \prime} \mathrm{O}$ \\
\hline PT 31 & Rio Piabanha & SM & $13^{\circ} 15^{\prime} 34,4^{\prime \prime} \mathrm{S}$ e $52^{\circ} 09^{\prime} 00,5^{\prime \prime} \mathrm{O}$ \\
\hline PT 32 & Rio Suiá-Miçu ponto 1 & SM & $13^{\circ} 15^{\prime} 45,5^{\prime \prime} \mathrm{S}$ e $52^{\circ} 02^{\prime} 50,9^{\prime \prime} \mathrm{O}$ \\
\hline PT 33 & Rio Suiá-Miçu ponto 2 & SM & $13^{\circ} 15^{\prime} 24,3^{\prime \prime} \mathrm{S}$ e $52^{\circ} 08^{\prime} 44,5^{\prime \prime} \mathrm{O}$ \\
\hline PT 34 & Rio Suiá-Miçu ponto 3 & SM & $11^{\circ} 50^{\prime} 17,8^{\prime \prime} \mathrm{S}$ e $52^{\circ} 15^{\prime} 07,5^{\prime \prime} \mathrm{O}$ \\
\hline PT 35 & Rio Suiazinho & SM & $12^{\circ} 38^{\prime} 33,4^{\prime \prime} \mathrm{S}$ e $51^{\circ} 56^{\prime} 50,7^{\prime \prime} \mathrm{O}$ \\
\hline PT 36 & Córrego Taquaral nascente & $\mathrm{RP}$ & $15^{\circ} 44^{\prime} 20,4^{\prime \prime} \mathrm{S}$ e $52^{\circ} 23^{\prime} 31,3^{\prime \prime} \mathrm{O}$ \\
\hline PT 37 & Rio Pindaiba degradado & $\mathrm{RP}$ & $14^{\circ} 53^{\prime} 29,2^{\prime \prime} \mathrm{S}$ e $52^{\circ} 00^{\prime} 19,4^{\prime \prime} \mathrm{O}$ \\
\hline PT 38 & Córrego Taquaral degradado & $\mathrm{RP}$ & $15^{\circ} 41^{\prime} 24.9^{\prime \prime} \mathrm{S}$ e $52^{\circ} 18^{\prime} 37,0^{\prime \prime} \mathrm{O}$ \\
\hline PT 39 & Córrego Colher-foz & $\mathrm{RM}$ & $14^{\circ} 39^{\prime} 19,2^{\prime \prime} \mathrm{S}$ e $52^{\circ} 27^{\prime} 45,1^{\prime \prime} \mathrm{O}$ \\
\hline PT 40 & Córrego Colher-intermediário & $\mathrm{RM}$ & $14^{\circ} 38^{\prime} 1,8^{\prime \prime} \mathrm{S}$ e $52^{\circ} 26^{\prime} 38,5^{\prime \prime} \mathrm{O}$ \\
\hline PT 41 & Córrego Santo Antônio-nascente & $\mathrm{RM}$ & $14^{\circ} 36^{\prime} 36,6^{\prime \prime} \mathrm{S}$ e $52^{\circ} 29^{\prime} 13,8^{\prime \prime} \mathrm{O}$ \\
\hline PT 42 & Córrego Santo Antônio-foz & $\mathrm{RM}$ & $14^{\circ} 38^{\prime} 41,3^{\prime \prime} \mathrm{S}$ e $52^{\circ} 29^{\prime} 23,9^{\prime \prime} \mathrm{O}$ \\
\hline
\end{tabular}

RM = Rio das Mortes; RP = Rio Pindaíba; SM = Rio Suiá-Miçu.

Suiá-Miçú, foram encontrados indivíduos em ambientes alterados por represamento, em ambientes semi-lóticos e lóticos de pequeno porte.

Comentários: Esta espécie já foi registrada para o Mato Grosso por Salles et al. (2004b).

Material examinado: PT02: (4) 12.vii.2005; PT02: (4) 13.i.2005; PT03: (16) 13.i.2005; PT03: (1) 12.vii.2005; PT06: (1) 04.v.2007; PT07: (3) 03.xi.2007; PT07: (5) 04.viii.2007; PT10: (1) 02.xi.2007;
PT10: (1) 09.i.2005; PT10: (1) 25.x.2005; PT10: (2) 09.viii.2005; PT11: (10) 13.vii.2005; PT11: (1) 01.viii.2007; PT11: (1) 18.i.2008; PT11: (1) 25.x.2005; PT11: (2) 05.xi.2007; PT11: (3) 12.i.2005; PT11: (4) 05.v.2008; PT12: (11) 12.i.2005; PT12: (15) 16.vii.2005; PT12: (4) 05.v.2008; PT13: (2) 16.i.2005; PT13: (34) 14.vii.2005; PT13: (8) 20.xi.2005; PT14: (5) 07.i.2005; PT14: (5) 20.xi.2005; PT14: (7) 14.vii.2005; PT15: (1) 08.i.2005; PT15: (2) 19.xi.2005; 
PT15: (9) 19.vii.2005; PT16: (1) 08.i.2005; PT17: (4) 15.i.2005; PT17: (6) 15.vii.2005; PT17: (9) 20.x.2005; PT18: (5) 20.x.2005; PT19: (2) 18.vii.2005; PT19: (3) 19.i.2005; PT20: (1) 19.i.2005; PT21: (2) 28.i.2005; PT22: (1) 20.i.2005; PT26: (2) 25.ix.2007; PT26: (7) 19.xi.2005; PT29:(5) 17.xii.2007; PT29:(3) 27.v.2008; PT32: (1) 23.ix.2007; PT33: (1) 23.ix.2007.

Apobaetis fiuzai Salles \& Lugo-Ortiz, 2002

Distribuição: ES, MG, MT, RJ e SP.

Aspectos biológicos: Coletados em ambientes de $3^{\mathrm{a}}$ a $5^{\mathrm{a}}$ ordem na Bacia do Rio Pindaíba. Na Bacia do Rio Suiá-Miçú foi coletado apenas um indivíduo na margem de um rio de larga extensão, com aproximadamente $60 \mathrm{~m}$ de largura. $\mathrm{O}$ substrato desse ambiente era composto por silte, pedras e pouca matéria orgânica acumulada.

Comentários: Esta espécie já foi registrada para o estado por Salles \& Lugo-Ortiz (2002).

Material examinado: PT03: (1) 19.x.2005; PT04: (1) 11.vii.2005; PT04: (9) 19.x.2005; PT07: (1) 19.i.2008; PT08: (1) 20.i.2008; PT08: (1) 3.v.2008; PT08: (1) 3.xi.2007; PT11: (1) 01.viii.2007; PT15: (1) 19.vii.2005; PT16: (3) 19.vii.2005; PT20: (1) 18.vii.2005; PT20: (3) 21.x.2005; PT21: (2) 20.i.2005; PT34: (1) 18.xii.2007.

Aturbina maculata Salles, Boldrini \& Shimano, 2010

Distribuição: AM, MT e RO.

Aspectos biológicos: Esta espécie ocorreu em trechos de $1^{\mathrm{a}}$ a $5^{\mathrm{a}}$ ordem da Bacia do Rio Pindaíba, e em ambientes lóticos na Bacia do Suiá-Miçú.

Comentário: Esta espécie foi descrita recentemente por Salles et al. (2010a)

Material examinado: PT02: (1) 13.i.2005; PT03: (13) 19.x.2005; PT11: (11) 01.viii.2007; PT11: (4) 05.xi.2007; PT11: (8) 25.x.2005; PT12: (2) 01.viii.2007; PT15: (1) 19.vii.2005; PT16: (2) 19.xi.2005; PT17: (4) 10.x.2005; PT18: (1) 20.x.2005; PT19: (2) 21.x.2005; PT20: (1) 21.x.2005; PT21: (1) 26.x.2005; PT22: (1) 20.i.2005; PT25: (1) 27.ix.2007; PT31: (1) 20.xii.2007; PT31: (2) 23.ix.2007; PT31: (2) 24.v.2008; PT32: (1) 20.xii.2007; PT32: (2) 23.ix.2007; PT33: (1) 26.v.2008; PT33: (3) 18.xii.2007; PT34: (3) 16.xii.200; PT34: (3) 25.v.2008.

Aturbina georgei Lugo-Ortiz \& McCafferty, 1996

Distribuição: AC, AM, BA, ES, MG, MT, RJ e PA.

Aspectos biológicos: Amplamente distribuída, esta espécie ocorreu em trechos de $1^{\mathrm{a}}$ a $6^{\mathrm{a}}$ ordem na Bacia do Rio Pindaíba, e no Rio Suiá-Miçú em águas lóticas e semi-lóticas.

Comentário: Aturbina georgei foi registrada anteriormente para o Mato Grosso por Lugo-Ortiz \& McCafferty (1996), Nolte et al. (1997) e Salles et al. (2004b).

Material examinado: PT01: (1) 12.vii.2005; PT02: (19) 12.vii.2005; PT02: (2) 13.i.2005; PT03: (1) 12.vii.2005; PT03: (8) 19.x.2005; PT04: (28) 19.x. 2005; PT11: (19) 25.x.2005; PT11: (20) 13.vii.2005; PT11: (7) 12.i.2005; PT12: (1) 17.xi.2005; PT12: (3) 16.vii.2005; PT14: (1) 20.xi.2005; PT14: (2) 07.i.2005; PT15: (2) 19.vii.2005; PT15: (3) 19.xi.2005; PT16: (6) 19.xi.2005; PT17: (11) 20.x.2005; PT17: (2) 15.i.2005; PT17: (3) 15.vii.2005; PT18: (1) 20.x.2005; PT18: (2) 15.i.2005; PT19: (3) 21.x.2005; PT20: (1) 18.vii.2005; PT20: (3) 21.x.2005; PT20: (5) 19.i.2005; PT21: (1) 26.x. 2005; PT22: (2) 20.i.2005; PT23: (1) 04.ix.2005; PT26: (1) 18.xii.2007; PT26: (3) 25.ix.2007; PT30: (1) 27.v.2008; PT30: (3) 26.ix.2007; РT30: (8) 17.xii.2007; PT33: (1) 23.ix.2007; PT34: (1) 18.xii.2007; PT34: (1) 25.ix.2007; PT34: (1) 26.x.2008.

Baetodes sp.

Aspectos biológicos: A espécie não-identificada de Baetodes foi amostrada em ambientes de $1^{\mathrm{a}}$ a $3^{\mathrm{a}}$ ordem em córregos considerados preservados e não foi amostrado na Bacia do Rio Suiá-Miçú.
Comentários: Essa morfoespécie não foi identificada pois se trata provavelmente de uma espécie nova. Esse é o primeiro registro do gênero para o estado.

Material examinado: PT09: (1) 02.viii.2007; PT09: (1) 09.i.2005; PT09: (1) 24.x.2005; PT09: (4) 17.i.2008; PT11: (4) 05.v.2008; PT13: (1) 15.7.2005; PT13: (1) 15.i.2005; PT13: (1) 16.i.2005; PT13: (6) 14.vii.2005; PT15: (2) 08.i.2005; PT15: (2) 08.i.2005; PT18: (3) 15.vii.2005.

\section{Callibaetis sp.1}

Aspectos biológicos: Essa morfoespécie foi bem distribuída na Bacia do Rio Pindaíba, ocorrendo em córregos de $1^{\mathrm{a}}$ a $5^{\mathrm{a}}$ ordem, enquanto que na Bacia do Rio Suiá-Miçú, ocorreu apenas em córregos semi-lóticos (PT24, PT26 e PT30).

Comentários: Essa morfoespécie, assim como as demais do gênero Callibaetis, não pôde ser identificada a nível específico uma vez que a maioria das espécies desse gênero foram descritas apenas pelos adultos. Essa morfoespécie se diferencia das demais por possuir dentículos em todas as garras tarsais e ter uma escavação média no labro.

Material examinado: PT02: (3) 13.i.2005, PT02: (5) 12.vii.2005; PT03: (1) 10.x.2005; PT04: (3) 19.x.2005; PT12: (2) 16.vii.2005; PT15: (2) 19.vii.2005; PT16: (1) 19.vii.2005; PT16: (1) 19.xi.2005; PT17: (1) 20.x.2005; PT18: (1) 20.x.2005; PT18: (7) 15.vii.2005; PT20: (2) 19.i.2005; PT20: (7) 21.x.2005; PT21: (4) 16.vii.2005; PT24: (3) 25.v.2008; PT26: (1) 18.xii.2007; PT30: (5) 27.v.2008; PT30: (9) 17.xii.2007; РT30: (9) 26.ix.2007.

\section{Callibaetis sp. 2}

Aspectos biológicos: Apenas um indivíduo dessa espécie foi encontrado em um córrego com mata ciliar alterada na Bacia do Rio Pindaíba. Já na Bacia do Rio Suiá-Miçú, essa espécie foi abundante, aparecendo em trechos de águas lóticas e matas ciliares preservadas até córregos represados sem vegetação ribeirinha.

Comentários: Essa morfoespécie se diferenciou das demais morfoespécies devido suas garras posteriores obterem apenas microdentículos, que não são visíveis a olho nu, enquanto que a garra mediana possui dentículos visíveis, e ainda, por apresentarem uma escavação pequena no labro.

Material examinado: PT02: (1) 12.vii.2005; PT24: (10) 16.xii.2007; PT24: (21) 25.v.2008; PT24: (3) 24.ix.2007; PT25: (1) 23.v.2008; PT26: (12) 18.xii.2007; PT26: (6) 26.v.2008; PT26: (7) 25.ix.2007; PT27: (3) 27.ix.2007; PT27: (6) 23.v.2008; PT29: (4) 27.v.2008; PT29: (7) 17.xii.2007; PT30: (6) 17.xii. 2007; PT30: (6) 26.ix. 2007; PT35: (1) 25.v.2008.

\section{Callibaetis sp. 3}

Aspectos biológicos: Esse morfótipo foi o menos abundante do gênero, sendo encontrado apenas na Bacia do Rio Pindaíba, em trechos de $2^{\mathrm{a}}$ a $4^{\mathrm{a}}$ ordens.

Comentários: Essa morfoespécie se diferenciou das demais devido as garras medianas e posteriores não possuírem dentículos, apresentar o labro mais arrendondado lateralmente e uma escavação grande.

Material examinado: PT03: (3) 19.x.2005; PT11: (1) 13.vii.2005; PT14: (1) 20.xi.2005; PT15: (2) 19.vii.2005; PT16: (1) 19.vii.2005; PT16: (1) 19.xi.2005.

Camelobaetidius janae Dominique \& Thomas, 2000

Distribuição: MT e RO.

Aspectos biológicos: Foram coletados indivíduos em trechos de $2^{\mathrm{a}}$ a $4^{\mathrm{a}}$ ordem da Bacia do Rio Pindaíba, em diversos tipos de substratos, com exceção de areia. Esta espécie não foi amostrada na Bacia do Rio Suiá-Miçú. 
Comentários: A espécie foi registrada para o estado por Salles et al. (2004b) e Salles \& Serrão (2005).

Material examinado: PT02: (3) 13.i.2005; PT03: (7) 13.i.2005; PT11: (1) 13.vii.2005; PT12: (1) 12.i.2005; PT15: (2) 19.xi.2005; PT16: (5) 19.xi.2005; PT19: (4) 19.i.2005; PT22: (1) 16.vii.2005.

Cloeodes auwe Salles \& Batista, 2004

\section{Distribuição: MT.}

Aspectos biológicos: Foram coletados indivíduos em ambientes de $1^{\mathrm{a}}, 2^{\mathrm{a}}, 5^{\mathrm{a}}$ e $6^{\mathrm{a}}$ ordens na Bacia do Rio Pindaíba. No Rio Suiá-Miçú a espécie foi coletada apenas em ambientes lóticos de pequeno e médio porte.

Comentários: Cloeodes auwe já havia sido registrada para o estado por Salles et al. (2004b).

Material examinado: PT04: (2) 19.x.2005; PT05: (1) 06.v.2008; PT08: (2) 03.xi.2007; PT08: (2) 06.viii.2007; PT09: (11) 05.xi.2007; PT09: (1) 21.vii.2005; PT09: (6) 03.viii.2007; PT21: (1) 26.x.2005; PT22: (2) 20.i.2005; PT32: (2) 23.ix.2007; PT32: (2) 24.v.2008; PT35: (7) 16.xii.2007.

Cloeodes hydation McCafferty \& Lugo-Ortiz, 1995

Distribuição: ES, MG e MT.

Aspectos biológicos: Esta espécie foi coletada principalmente em trechos preservados de $1^{\mathrm{a}}$ a $3^{\mathrm{a}}$ ordem na Bacia do Rio Pindaíba e não foi amostrada na Bacia do Rio Suiá-Miçú.

Comentários: A espécie já foi registrada para o estado por McCafferty \& Lugo-Ortiz (1995).

Material examinado: PT02: (1) 12.vii.2005; PT03: (2) 19.x.2005; PT11: (1) 13.vii.2005; PT17: (1) 20.x.2005; PT17: (2) 15.vii.2005; PT18: (13) 20.x.2005; PT18: (6) 15.vii.2005.

Cloeode redactus Watz \& McCafferty, 1987

Distribuição: MT.

Aspectos biológicos: Coletados em ambientes de $1^{\mathrm{a}}$ a $4^{\mathrm{a}}$ ordem na Bacia do Rio Pindaíba, esta espécie foi bem representada. Apenas um indivíduo foi amostrado no Rio Suiá-Miçú.

Comentários: Este é o primeiro registro desta espécie para o Brasil. Anteriormente sua distribuição estava restrita para Colômbia e Peru (Domínguez et al. 2006).

Material examinado: PT02: (2) 13.i.2005; PT02: (4) 12.vii.2005; PT04: (4) 19.x.2005; PT07: (7) 19.x.2005; PT09: (1) 21.vii.2005; PT09: (1) 24.x.2005; PT10: (2) 25.x.2005; PT11: (12) 25.x.2005; PT12: (1) 16.vii.2005; PT12: (1) 17.xi.2005; PT14: (1) 07.i.2005; PT16: (1) 19.vii.2005; PT16: (2) 19.xi.2005; PT17: (3) 15.vii.2005; PT17: (43) 20.x.2005; PT18: (11) 15.vii.2005; PT18: (6) 20.x.2005; PT18: (8) 15.i.2005; PT19: (1) 21.x.2005; PT20: (15) 21.x.2005; PT20: (1) 18.vii.2005; PT32: (1) 23.ix.2007.

\section{Cryptonympha copiosa Lugo-Ortiz \& McCarfferty, 1998}

Distribuição: AC, AM, MT, PA, RS e SC.

Aspectos biológicos: Coletados em ambientes de $2^{\mathrm{a}}$ a $4^{\mathrm{a}}$ ordem na Bacia do Rio Pindaíba, sendo mais abundante na Bacia do Rio Suiá-Miçú, onde foi coletado em vários tipos de ambientes, como semi-lóticos, lóticos e alterados.

Comentários: Esse é o primeiro registro do gênero e espécie para o Estado do Mato Grosso.

Material examinado: PT03: (1) 13.i.2005; PT04: (1)19.x.2005; PT06: (1) 04.viii.2007; PT06: (1) 19.i.2008; PT06: (3) 19.i.2008; PT11: (1) 01.viii.2007; PT11: (2) 18.i.2008; PT21: (3) 28.i.2005; PT24: (10) 25.v.2008; PT24: (2) 24.ix.2007; PT24:(7) 16.xii.2007; PT25: (2) 19.xii.2007; PT26: (1) 26.v.2008; PT26: (2) 18.xii.2007; PT26: (4) 25.ix.2007; PT27: (1) 27.ix.2007; PT27: (8) 23.v.2008; PT29: (1) 27.v.2008; PT29: (1) 28.ix.2007; PT30: (4N) 17.xii.2007; PT33: (1) 20.xii.2007; PT35: (1) 16.xii.2007; PT35: (1) 25.v.2008.
Guajirolus rondoni Salles, 2007

Distribuição: MT e RO.

Aspectos biológicos: Coletado apenas um indivíduo na Bacia do Pindaíba, na $3^{\mathrm{a}}$ ordem de um córrego preservado. Foi coletado associado ao substrato folhiço de remanso.

Comentários: Esse é o primeiro registro do gênero e espécie para o Estado do Mato Grosso.

Material examinado: PT15: (1) 19.vii.2005.

Harpagobaetis gulosus Mol, 1986

Distribuição: GO e MT.

Aspectos biológicos: Foram coletados apenas dois indivíduos desta espécie, um na Bacia do Rio Pindaíba, em um córrego de $3^{\text {a }}$ ordem com alto grau de conservação, associado ao substrato madeira; e um na Bacia do Rio Suiá-Miçú, também em um local altamente conservado.

Comentários: Esta espécie já havia sido registrada para o estado por Salles et al. (2004b).

Material examinado: PT11: (1) 12.i.2005; PT25: (1) 23.v.2008.

Paracloeodes binodulus Lugo-Ortiz \& McCafferty, 1996

Distribuição: AM, MT e PA.

Aspectos biológicos: Coletados em ambientes de $2^{\mathrm{a}}$ a $4^{\mathrm{a}}$ ordens em córregos relativamente conservados ou alterados na Bacia do Rio Pindaíba, e apenas em um local na Bacia do Rio Suiá-Miçú, lótico de médio porte, relativamente alterado pela pecuária.

Comentários: Esta espécie já havia sido registrada para o Mato Grosso por Salles et al. (2004b).

Material examinado: PT02: (1) 13.i.2005; PT03: (2) 13.i.2005; PT03: (1) 19.x.2005; PT12: (2) 16.vii.2005; PT12: (1) 17.xi.2005; PT15: (1) 08.i.2005; PT16: (14) 19.xi.2005; PT35: (2) 16.xii.2007.

Spiritiops silvudus Lugo-Ortiz \& McCafferty, 1998

Distribuição: AM, BA, MT e PA.

Aspectos biológicos: Amostrado em coletas avulsas, na nascente do Córrego Taquaral, Bacia do Rio Pindaíba, em trechos conservados e em trechos impactado do Rio Pindaíba.

Comentários: Esta espécie foi registrada anteriormente por Salles et al. (2004b).

Material examinado: PT36: (6) 05.iv.2004; PT37: (1) 03.iv.2004.

Varipes helenae Salles \& Batista, 2004

Distribuição: MT.

Aspectos biológicos: Esta espécie não foi amostrada em nenhuma das coletas realizadas, porém, foi amostrada em 2004 em coletas avulsas no Rio Pindaíba e no Córrego Taquaral, em trechos impactados, compondo a série tipo da descrição de Salles \& Batista (2004).

Comentários: Esta espécie foi anteriormente registrada para o Mato Grosso por Salles \& Batista (2004).

Waltzoyphius fasciatus McCafferty \& Lugo-Ortiz, 1995

Distribuição: AM, BA, ES, MG, MT, PA, RJ e SP.

Aspectos biológicos: Espécie amplamente distribuída na Bacia do Rio Pindaíba em trechos de $1^{\mathrm{a}}$ a $4^{\mathrm{a}}$ ordens, porém não encontrada na Bacia do Rio Suiá-Miçú.

Comentários: Waltzoyphius fasciatus já havia sido registrada para o estado por Salles et al. (2004b).

Material examinado: PT02: (2) 12.vii.2005; PT02: (4) 13.i.2005; PT03: (2) 12.vii.2005; PT03: (2) 19.x.2005; PT04: (3) 20.xi.2005; PT09: (1) 02.viii.2007; PT09: (3) 09.i.2005; PT10: (1) 02.xi.2007; PT10: (1) 09.viii.2005; PT10: (2) 03.viii.2007; PT11: (10) 05.xi.2007; PT11: (10) 25.x.2005; PT11: (1) 01.viii.2007; PT11: (1) 18.i.2008; PT11: (2) 12.i.2005; PT11: (6) 13.vii.2005; PT12: (3) 
17.xi.2005; PT12: (43) 16.vii.2005; PT12: (9) 12.i.2005; PT14: (6) 14.vii.2005; PT14: (7) 07.i.2005; PT14: (9) 20.xi.2005; PT15: (2) 19.xi.2005; PT16: (1) 19.vii.2005; PT16: (2) 19.xi.2005; PT18: (6) 15.vii.2005; PT19: (1) 19.i.2005.

Waltzoyphius roberti Thomas \& Peru, 2002

\section{Distribuição: MT.}

Aspectos biológicos: Amplamente distribuída na Bacia do Rio Pindaíba, em trechos de $1^{\text {a }}$ a $5^{\text {a }}$ ordens. Na Bacia do Rio Suiá-Miçú também foi amplamente distribuída, estando presente em ambientes lóticos, semi-lóticos e alterados/represados.

Comentários: Primeiro registro da espécie para o Brasil e consequentemente para o Estado do Mato Grosso. De acordo com Domínguez et al. (2006) há registro desta espécie apenas para a Guiana Francesa. Material examinado: PT02: (2) 13.i.2005; PT02: (4) 12.vii.2005; PT03: (1) 13.i.2005; PT03: (5) 19.x.2005; PT03: (7) 12.vii.2005; PT04: (1) 19.x.2005; PT05: (1) 03.xi.2007; PT05: (1) 04.viii.2007; PT05: (1) 19.i.2008; PT05: (4) 03.viii.2007; PT06: (1) 03.xi.2007; PT06: (1) 06.viii.2007; PT09: (5) 09.i.2005; PT10: (1) 02.xi.2007; PT10: (1) 03.viii.2007; PT10: (2) 25.x.2005; PT10: (6) 09.viii.2005; PT11: (1) 12.i.2005; PT11: (1) 13.vii.2005; PT12: (1) 16.vii.2005; PT12: (2) 17.xi.2005; PT12: (2) 21.x.2005; PT14: (1) 20.xi.2005; PT14: (21) 19.xi.2005; PT14: (25) 19.vii.2005; PT14: (2) 19.vii.2005; PT14: (5) 08.i.2005; PT14: (7) 07.i.2005; PT19: (1) 19.i.2005; PT19: (2) 21.x.2005; PT19: (4) 18.vii.2005; PT20: (2) 21.10.2005; PT20: (5) 18.vii.2005; PT21: (2) 28.i.2005; PT24: (3) 24.ix.2007; PT24: (4) 25.v.2008; PT25: (1) 19.xii.2007; PT25: (4) 23.v.2008; PT26: (1) 18.xii.2007; PT27: (1) 27.ix.2007; PT32: (1) 24.v.2008; PT33: (1) 23.ix.2007; PT34: (1) 18.xii.2007; PT35: (1) 16.xii.2007; PT35: (2) 25.v.2008.

Zelusia principalis Lugo-Ortiz \& McCafferty, 1998

Distribuição: AM, BA, MG, MT, PA, RJ e SP.

Aspectos biológicos: Esta espécie se apresentou abundante na Bacia do Rio Pindaíba, indivíduos foram coletados em trechos de $1^{\text {a a }} 5^{\mathrm{a}}$ ordem. Já na Bacia do Rio Suiá-Miçú foi pouco abundante e apareceu somente em ambientes lóticos de pequeno e médio porte.

Comentários: Esta espécie já foi registrada para o Mato Grosso por Salles et al. (2004b).

Material examinado: PT02: (32) 12.vii.2005; PT02: (8) 13.i.2005; PT03: (13) 19.x.2005; PT03: (8) 12.vii.2005; PT03: (8) 13.i.2005; PT04: (2) 19.x.2005; PT04: (4) 11.vii.2005; PT09: (11) 09.i.2005; PT09: (13) 02.viii.2007; PT09: (19) 24.x.2005; PT09: (3) 02.xi.2007; PT09: (3) 17.i.2008; PT09: (5) 11.vii.2005; PT09: (5) 17.i.2005; PT10: (17) 03.viii.2007; PT10: (1) 04.v.2008; PT10: (1) 17.i.2008; PT10: (21) 25.x.2005; PT10: (2) 02.xi.2007; PT10: (77) 09.vii.2005; PT11: (2) 05.v.2008; PT11: (2) 18.i.2008; PT11: (32) 12.i.2005; PT11: (5) 01.viii.2007; PT11: (6) 05.xi.2007; PT11: (7) 13.vii.2005; PT11: (7) 25.x.2005; PT12: (1) 01.viii.2007; PT12: (1) 05.v.2008; PT12: (22) 16.vii.2005; PT12: (5) 17.xi.2005; PT12: (7) 12.i.2005; PT13: (11) 14.vii.2005; PT13: (11) 20.xi.2005; PT13: (1) 20.xi.2005; PT13: (2) 07.i.2005; PT13: (2) 14.vii.2005; PT14: (6) 19.xi.2005; PT14: (8) 19.vii.2005; PT15: (16) 19.xi.2005; PT15: (4) 19.vii.2005; PT17: (101) 20.x.2005; PT17: (22) 15.vii.2005; PT17: (4) 15.i.2005; PT18: (3) 15.i.2005; PT18: (4) 15.vii.2005; PT18: (5) 20.x.2005; PT19: (11) 21.x.2005; PT19: (17) 18.vii.2005; PT19: (22) 19.i.2005; PT20: (13) 21.vii.2005; PT20: (16) 18.vii.2005; PT20: (30) 19.i.2005; PT21: (1) 28.i.2005; PT21: (4) 16.vii.2005; PT31: (1) 23.ix.2007; PT32: (24) 23.ix.2007; PT33: (1) 23.ix.2007; PT35: (1) 16.xii.2007.

\section{Caenidae}

Caenidae foi estabelecido como uma tribo (Caenini) por Banks em 1900, e em 1909 foi elevada à família por Klapálek. Esta família ocorre em uma variedade de lagos, lagoas, córregos e rios sobre toda a Terra, sendo ausente apenas na Nova Zelândia e na maioria das ilhas oceânicas (Edmunds Junior et al. 1976).

As ninfas são encontradas em praticamente todos os tipos de ambiente aquáticos, geralmente preferindo águas lênticas como lagos, lagoas e remansos de córregos e rios, onde ocorre deposição de detritos (McCafferty et al. 1997, Salles 2006). Também podem ser encontradas agregadas à vegetação em zonas de correntezas e em macrófitas flutuantes em ambientes lênticos. São organismos tolerantes a uma ampla mudança na temperatura e variação no nível de poluição (Domínguez et al. 2006).

Na América do Sul os Caenidae são representados por quatro gêneros, sendo Brasilocaenis Puthz, 1975 e Caenis Stephens, 1835 mais diversos, enquanto que Brachycercus Curtis, 1834 e Cercobrachys Soldán, 1986 são raros (Molineri \& Malzacher 2007). No Brasil não há registro de Brachycercus e no Mato Grosso, havia apenas o registro do gênero Brasilocaenis (Salles 2010).

\section{Brasilocaenis irmeli Puthz, 1975}

Distribuição: AM e MT.

Aspectos biológicos: Coletado apenas na Bacia do Rio SuiáMiçú, em dois locais, um degradado e próximo a uma represa, e outro em um rio de grande porte com aproximadamente $60 \mathrm{~m}$ de largura.

Comentários: Esta espécie já foi registrada para o estado por Malzacher (1998).

Material examinado: PT28: (1) 27.v.2008; PT34: (4) 26.v.2008.

Caenis cuniana Froehlich, 1969

Distribuição: MT, ES, RJ e SP.

Aspectos biológicos: Coletados apenas em trechos alterados de $1^{\mathrm{a}}$ e $2^{\mathrm{a}}$ ordens na Bacia do Rio Pindaíba e na Bacia do Rio Suiá-Miçú, em córregos e rios de águas semi-lóticas com muito material orgânico em decomposição e em ambientes de águas lênticas represadas/alteradas.

Comentários: Esse é o primeiro registro da espécie para o Estado do Mato Grosso.

Material examinado: PT05: (1) 06.v.2008; PT05: (5) 03.viii.2007; PT24: (12) 25.v.2008; PT24: (1) 24.ix.2007; PT24: (60) 16.xii.2007; PT26: (13) 26.v.2008; PT26: (24) 18.xii.2007; PT26: (8) 25.ix.2007; PT27: (2) 27.ix.2007; PT27: (8) 23.v.2008; PT28: (4) 17.xii.2007; PT29: (5) 27.v.2008; PT29: (7) 28.ix.2007; PT29: (8) 17.xii.2007; РT30: (3) 17.xii.2007.

Caenis fittkaui Malzacher, 1986

Distribuição: ES, MT e PA.

Aspectos biológicos: Coletados apenas na Bacia do Rio SuiáMiçú, em ambientes de águas semi-lóticas com muito material orgânico em decomposição e em águas represadas.

Comentários: Esse é o primeiro registro de C. fittkaui para o Mato Grosso.

Material examinado: PT24: (3) 16.xii.2007; PT26: (14) 18.xii.2007; PT26: (4) 25.ix.2007; PT26: (5) 26.v.2008; PT29: (1) 17.xii.2007; РT29: (1) 27.v.2008; PT29: (1) 27.v.2008; PT29: (2) 28.ix.2007; PT30: (1) 17.vii.2007.

\section{Caenis pflugfelderi Malzacher, 1990}

Distribuição: AM e MT.

Aspectos biológicos: Amostradas em ambientes de $1^{\mathrm{a}}$ a $5^{\mathrm{a}}$ ordem dos córregos e rios da Bacia do Rio Pindaíba, esta espécie foi a mais abundante e bem distribuída entre os Caenis. Na Bacia do Rio SuiáMiçu, também foi a espécie menos exigente quanto ao tipo de hábitat, sendo encontrada em ambientes lóticos e alterados.

Comentários: Esse é o primeiro registro da espécie para o Estado do Mato Grosso. 
Material examinado: PT01: (7) 12.vii.2005; PT02: (1) 12.vii.2005; PT03: (1) 19.x.2005; PT05: (6) 03.viii.2007; PT06: (1) 04.viii.2007; PT06: (8) 06.v.2008; PT07: (2) 03.v.2008; PT08: (1) 06.viii.2007; PT08: (6) 03.xi.2007; РT09: (1) 18.i.2008; PT09: (2) 05.v.2008; PT13: (1) 20.xi.2005; PT15: (2) 29.vii.2005; PT16: (1) 19.xi.2005; PT16: (2) 19.vii.2005; PT17: (1) 15.vii.2005; PT17: (3) 15.i.2005; PT17: (3) 20.x.2005; PT18: (1) 15.vii.2005; PT18: (5) 20.x.2005; PT19: (2) 21.x.2005; PT21: (2) 28.i.2005; PT28: (3) 27.v.2008; PT30: (3) 27.v.2008; PT31: (2) 23.ix.2007; РT32: (1) 24.v.2008; РT33: (1) 23.ix.2007; РT34: (1) 25.ix.2007; РT34: (3) 26.v.2008; PT35: (1) 25.v.2008.

\section{Coryphoridae}

Esta família é monotípica e foi estabelecida por Molineri, Peters \& Cardoso em 2001. É grupo irmão da família Leptohyphidae (Dias et al. 2007b) e apresenta várias características únicas (Peters 1981). A sua biologia é pouco conhecida, as ninfas foram coletadas em córregos florestados das Bacias do Rio Amazonas e Orinoco (Colômbia), no norte do Brasil, Colômbia e Guiana Francesa (Dias et al. 2007b).

De acordo com Salles (2006), são encontradas exclusivamente em barrancos de rios, onde a velocidade da água é baixa, apresentando acúmulo de matéria orgânica fina depositada sobre o substrato. No Brasil há registro da família apenas para o Amazonas e Pará.

Coryphorus aquilus Peters, 1981

Distribuição: AM, MT e PA.

Aspectos biológicos: Esta espécie foi amostrada em apenas dois locais na Bacia do Rio Pindaíba, na $3^{\mathrm{a}}$ ordem do Córrego Cachoerinha (alterado) e $5^{\mathrm{a}}$ ordem do Rio Corrente. Na Bacia do Rio Suiá-Miçú foram coletados indivíduos em ambientes conservados (lóticos de pequeno e médio porte), alterados, ambientes represados e semilóticos, sempre em áreas de remanso, com acúmulo de matéria finamente particulada.

Comentários: C. aquilus é uma espécie típica da Bacia Amazônica, encontrada não só no Brasil, mas também em córregos amazônicos da Colômbia (Peters 1981). A Bacia do Rio Suiá-Miçú, também parte da Bacia Amazônica, se diferencia dos demais locais por estar em áreas de transição com o Cerrado, sendo esse o primeiro registro para o Estado do Mato Grosso.

Material examinado: PT03: (1) 19.x.2005; PT21: (1) 28.i.2005; PT21: (2) 16.vii.2005; PT25: (2) 19.xii.2007; PT25: (3) 23.v.2008; PT27: (1) 27.ix.2007; PT30: (1) 26.ix.2007; PT31: (1) 23.ix.2007; PT31: (1) 24.v.2008; PT31: (2) 20.xii.2007; PT33: (1) 16.xii.2007; PT33: (1) 23.ix.2007; PT33: (1) 24.v.2008.

\section{Euthyplociidae}

Esta família possui distribuição pantropical, e é dividida em duas subfamílias, Euthyplociinae, distribuída na America do Sul, Madagascar e Ásia, e Exeuthyplociinae, com distribuição restrita à África (Edmunds Junior et al. 1976, Domínguez et al. 2006).

Pouco se sabe a respeito da biologia desse grupo de insetos, apenas que indivíduos da subfamília Euthyplociinae são rastejadores, e escavam túneis horizontais no sedimento fino em baixo de grandes pedras, onde se protegem da correnteza (Edmunds Junior et al. 1976, Pereira \& Da-Silva 1990, Dominguez et al. 2006). As ninfas são encontradas exclusivamente em áreas de remansos de ambientes lóticos, ou protegidas da correnteza (Salles 2006).

De acordo com a lista de Salles (2010), no Brasil existem dois gêneros (Campylocia Needham \& Murphy, 1924 e Euthyplocia Eaton, 1871) e quatro espécies, sendo que nenhuma é registrada para o Estado do Mato Grosso.
Campylocia sp.

Aspectos biológicos: Amplamente distribuído, esse gênero foi coletado em ambientes de $1^{\mathrm{a}}$ a $5^{\mathrm{a}}$ ordem do Rio Pindaíba; no Rio Suiá-Miçú, foi presente apenas em córregos de pequeno a médio porte (até $16 \mathrm{~m}$ de largura).

Comentários: Esse é o primeiro registro do gênero para o estado.

Material examinado: PT03: (19) 12.vii.2005; PT03: (5) 19.x.2005; PT04: (1) 19.x.2005; PT09: (3) 02.viii.2007; PT09: (3) 21.vii.2005; PT10: (1) 25.x.2005; PT10: (2) 03.viii.2007; PT11: (1) 05.v.2008; PT11: (1) 12.i.2005; PT11: (1) 13.vii.2005; PT11: (3) 13.vii.2005; PT11: (6) 01.viii.2007; PT12: (10) 05.v.2008; PT12: (16) 01.viii.2007; PT12: (3) 12.i.2005; PT12: (4) 05.xi.2007; PT12: (8) 16.vii.2005; PT14: (1) 20.xi.2005; PT14: (2) 14.vii.2005; PT19: (1) 18.vii.2005; PT19: (4) 21.x.2005; PT20: (11) 21.x.2005; PT20: (13) 18.vii.2005; PT21: (2) 26.x.2005; PT21: (5) 16.vii.2005; PT25: (1) 27.ix.2007; PT25: (6) 23.v.2008; PT31:(1) 20.xii.2007; PT31:(1) 23.ix.2007; PT32: (5) 23.ix.2007; PT35: (1) 16.xii.2007; PT35: (1) 25.v.2008.

\section{Leptohyphidae}

Descrita primeiramente como uma subfamília (Leptohyphinae) de Tricorythidae, Leptohyphidae foi elevada ao nível de família em 1973 por Landa. Dentre os Ephemerelloidea, superfamília que abriga Coryphoridae e Melanemerellidae, além de outras famílias não ocorrentes na América do Sul, Leptohyphidae possui uma distribuição pan-americana e é a mais representativa no continente (Dias et al. 2007b).

Ninfas desta família são encontradas exclusivamente em ambientes lóticos, em diversos tipos de substratos, como pedra, cascalho, folhiço, barranco, hidrófita e áreas de deposição de matéria orgânica fina (Salles 2006). A presença de brânquias operculares facilita a tolerância a sólidos suspensos, e em córregos e rios muito turvos esta família torna-se um componente importante na comunidade bentônica (Domínguez et al. 2006).

No Brasil são registrados sete gêneros e 32 espécies (Salles 2010). No Mato Grosso pouco se tem estudado a respeito desta família, tendo sido realizado apenas um estudo com a descrição de uma espécie nova (Dias et al. 2009a).

Amanahyphes saguassu Salles \& Molineri, 2006

Distribuição: AM, MT e PA.

Aspectos biológicos: Coletado apenas na $5^{\mathrm{a}}$ ordem do Rio Corrente na Bacia do Rio Pindaíba, enquanto que na Bacia do Rio Suiá-Miçú esteve presente em córregos brejosos e lóticos de pequeno e médio porte.

Comentários: Esse é o primeiro registro do gênero e espécie para o Estado do Mato Grosso.

Material examinado: PT21: (2) 16.vii.2005; PT24: (1) 24.ix.2007; PT25:(1) 27.ix.2007; PT25:(5) 19.xii.2007; PT25:(6) 23.v.2008; PT31: (2) 24.v.2008; РT32: (2) 23.ix.2007; РT33: (1) 23.ix.2007; PT33: (6) 24.v.2008; PT35: (5) 25.v.2008.

Leptohyphes sp. nov.

Aspectos biológicos: Espécie amostrada apenas na Bacia do Rio Pindaíba, em trechos de $1^{\mathrm{a}}, 2^{\mathrm{a}}, 3^{\mathrm{a}}$ e $5^{\mathrm{a}}$ ordens.

Comentários: Esta morfoespécie apresentou um conjunto de características diferentes, como o labro coberto por cerdas plumosas (característico de Lehtohyphes liniti Wang, Sites \& McCafferty, 1998), porém com três a quatro dentículos submarginais subapicais nas garras anteriores, que deveriam ser ausentes nesta espécie. Trata-se, portanto, de uma possível espécie nova.

Material examinado: PT02: (1) 13.i.2005; PT09: (8) 09.i.2005; PT10: (1) 17.i.2008; PT10: (3) 02.xi.2007; PT10: (3) 09.viii.2005; 
PT10: (5) 04.v.2008; PT10: (6) 03.viii.2007; PT10: (8) 09.viii.2005; PT11: (1) 01.viii.2007; PT11: (1) 05.v.2008; PT11: (1) 12.i.2005; PT13: (24) 14.vii.2005; PT13: (33) 20.xi.2005; PT13: (41) 16.i.2005; PT17: (13) 15.i.2005; PT17: (29) 15.vii.2005; PT17: (6) 20.x.2005; PT18: (4) 15.vii.2005; PT18: (6) 20.x.2005; PT18: (7) 15.i.2005; PT21: (1) 16.vii.2005.

\section{Traverhyphes spp.}

Aspectos biológicos: Amplamente distribuído e muito abundante, esse gênero foi coletado em ambientes de $1^{\mathrm{a}}$ a $5^{\mathrm{a}}$ ordem na Bacia do Pindaíba e apenas em córregos de pequeno porte da mesma bacia.

Comentários: Traverhyphes não foi morfoespeciado devido à dificuldade taxonômica relacionada à fase imatura do gênero. É o primeiro registro do gênero para o estado.

Material examinado: PT01: (1) 13.i.2005; PT02: (11) 13.i.2005; PT02: (16) 12.vii.2005; PT03: (1) 13.i.2005; PT04: (1) 19.x.2005; PT04: (2) 11.vii.2005; PT04: (6) 18.i.2005; PT06: (1) 04.xi.2007; PT09: (16) 17.i.2008; PT09: (1) 21.vii.2005; PT09: (3) 01.ix.2005; PT09: (3) 02.viii.2007; PT09: (3) 04.v.2008; PT09: (4) 17.i.2005; PT09: (71) 09.i.2005; PT10: (18) 03.viii.2007; PT10: (27) 02.xi.2007; PT10: (30) 17.i.2008; PT10: (31) 09.i.2005; PT10: (41) 09.viii.2005; PT10: (42) 25.x.2005; PT10: (8) 04.v.2008; PT11: (14) 13.vii.2005; PT11: (15) 05.v.2008; PT11: (22) 01.viii.2007; PT11: (23) 05.xi.2007; PT11: (23) 05.xi.2007; PT11: (2) 23.x.2005; PT11: (32) 12.i.2005; PT11: (8) 18.i.2008; PT12: (106) 12.i.2005; PT12: (10) 17.xi.2005; PT12: (17) 16.vii.2005; PT12: (4) 05.xi.2007; PT12: (7) 05.v.2008; PT12: (8) 01.viii.2007; PT12: (8) 18.i.2008; PT13: (10) 20.xi.2005; PT13: (13) 14.vii.2005; PT13: (15) 16.i.2005; PT14: (27) 14.vii.2005; PT14: (49) 20.xi.2005; PT14: (6) 07.i.2005; PT15: (19) 08.i.2005; PT15: (42) 19.xi.2005; PT15: (6) 19.vii.2005; PT16: (32) 19.xi.2005; PT16: (3) 08.i.2005; PT17: (25) 15.vii.2005; PT17: (33) 20.x.2005; PT17: (34) 15.i.2005; PT18: (11) 20.x.2005; PT18: (4) 15.vii.2005; PT18: (73) 15.i.2005; PT19: (12) 19.i.2005; PT19: (2) 21.x.2005; PT19: (3) 18.vii.2005; PT20: (2) 21.x.2005; PT20: (3) 18.vii.2005; PT20: (56) 19.i.2005; PT22: (1) 15.xi.2005; PT22: (36) 04.ix.2005.

Tricorythodes barbus Allen, 1967

Distribuição: MT e SC.

Aspectos biológicos: Esta espécie não foi amostrada nas bacias estudadas, porém foi coletada no Córrego Colher, em 2005 durante coletas avulsas nos substratos areia, raiz e pedras.

Comentários: Esse é o primeiro registro da espécie para o Estado do Mato Grosso.

Material examinado: PT39: (3) 23.vii.2005; PT40: (1) 23.vii.2005.

Tricorythodes hiemalis Molineri, 2001

Distribuição: ES, divisa ES/MG e MT.

Aspectos biológicos: Espécie muito abundante, foi coletada em trechos de $1^{\mathrm{a}}$ a $5^{\mathrm{a}}$ ordem na Bacia do Rio Pindaíba, e no Rio SuiáMiçú em ambientes brejosos e lóticos.

Comentários: Esse é o primeiro registro da espécie para o Estado do Mato Grosso.

Material examinado: PT01: (2) 13.i.2005; PT03: (1) 12.vii.2005; PT03: (2) 19.x.2005; PT03: (4) 13.i.2005; PT04: (12) 19.x.2005; PT04: (3) 18.i.2005; PT04: (5) 11.vii.2005; PT07: (1) 03.v.2008; PT07: (1) 04.viii.2007; PT07: (1) 19.i.2008; PT08: (4) 03.v.2008; PT08: (4) 03.xi.2007; PT09: (1) 21.vii.2005; PT09: (8) 09.i.2005; PT10: (1) 02.xi.2007; PT10: (1) 03.viii.2007; PT10: (1) 09.i.2005; PT10: (1) 25.x.2005; PT10: (2) 09.xiii.2005; PT10: (3) 17.i.2008; PT11: (12) 01.viii.2007; PT11: (13) 18.i.2008; PT11: (15) 05.xi.2007; PT11: (29) 12.i.2005; PT11: (3) 13.vii.2005; PT11: (3) 25.x.2005; PT11: (4) 05.v.2008; PT12: (12) 05.xi.2007; PT12: (13) 12.i.2005;
PT12: (1) 17.xi.2005; PT12: (3) 01.viii.2007; PT12: (3) 05.v.2008; PT12: (4) 18.i.2008; PT12: (5) 16.vii.2005; PT18: (1) 15.vii.2005; PT19: (11) 21.x.2005; PT19: (16) 18.vii.2005; PT20: (4) 18.vii.2005; PT20: (5) 19.i.2005; PT20: (8) 21.x.2005; PT21: (1) 16.vii.2005; PT22: (1) 15.xi.2005; PT22: (1) 20.i.2005; PT22: (4) 04.ix.2005; PT24: (14) 24.ix.2007; PT24: (1) 25.v.2008; PT25: (1) 27.ix.2007; PT30: (13) 27.v.2005; PT30: (3) 26.ix.2007; PT30: (4) 17.xii.2007; PT31: (1) 24.v.2008; PT31: (2) 23.ix.2007; PT35: (1) 16.xii.2007.

\section{Tricorythodes mirca Molineri, 2002}

Distribuição: ES e MT.

Aspectos biológicos: Não amostrado nas coletas realizadas, porém foi encontrado em outros córregos do Mato Grosso durante coletas avulsas. Tricorythodes mirca foi coletado no Córrego Santo Antônio, Córrego Colher, e em uma área impactada da $5^{\mathrm{a}}$ ordem do Rio Pindaíba.

Comentários: Esse é o primeiro registro da espécie para o Estado do Mato Grosso.

Material examinado: PT37: (1) 03.iv.2004; PT39: (3) 21.xii.2004; PT39: (9) 23.vii.2005; PT40: (18) 23.vii.2005; PT40: (3) 20.xii.2004; PT41: (1) 20.xii.2004; PT41: (2) 19.xii.2004; PT42: (22) 27.vii.2005; PT42: (2) 21.xii.2004.

Tricorythodes sallesi Dias, Cabette \& Souza, 2009

\section{Distribuição: MT.}

Aspectos biológicos: Coletados apenas na Bacia do Rio Pindaíba em trechos de $2^{\mathrm{a}}$ à $5^{\mathrm{a}}$ ordem, principalmente em trechos com vegetação marginal preservada, alguns espécimes coletados fazem parte da sérietipo que deu origem à descrição dessa espécie por Dias et al. (2009a).

Comentários: Esta espécie já havia sido registrada para o Mato Grosso por Dias et al. (2009a).

Material examinado: PT12: (1) 05.xi.2007, PT14: (3) 14.vii.2005, PT19: (6) 18.vii.2005, PT20: (4) 18.vii.2005, PT20: (9) 21.x.2005, PT21: (1) 16.vii.2005.

\section{Tricorythodes santarita Traver, 1959}

Distribuição: MT e RJ.

Aspectos biológicos: Foram coletados indivíduos em trechos de $1^{\mathrm{a}}$ a $4^{\mathrm{a}}$ ordem de córregos considerados conservados na Bacia do Rio Pindaíba, e não foram encontrados na Bacia do Rio Suiá-Miçú.

Comentários: Esse é o primeiro registro da espécie para o Estado do Mato Grosso.

Material examinado: PT09: (1) 09.i.2005; PT09: (2) 17.i.2005; PT10: (1) 03.viii.2007; PT10: (1) 09.viii.2005; PT12: (1) 05.xi.2007; PT12: (1) 17.xi.2005; PT15: (15) 19.xi.2005; PT15: (4) 19.vii.2005; PT16: (1) 19.xi.2005; PT19: (1) 18.vii.2005; PT19: (3) 21.x.2005; PT20: (1) 18.vii.2005.

Tricorythodes rondoniensis Dias, Cruz \& Ferreira, 2009

Distribuição: RO, RR e MT.

Aspectos biológicos: Foram amostrados poucos indivíduos apenas na Bacia do Rio Suiá-Miçú, em ambientes represados, semilóticos e lóticos.

Comentários: Esse é o primeiro registro da espécie para o Estado do Mato Grosso.

Material examinado: PT28: (1) 28.ix.2007; PT30: (1) 17.xii.2007; PT30: (1) 24.v.2008; PT30: (1) 26.ix.2007; PT35: (2) 16.xii. 2007 .

\section{Tricorythodes yura Molineri, 2002}

\section{Distribuição: ES e MT.}

Aspectos biológicos: Coletados apenas na Bacia do Rio Pindaíba, esta espécie foi presente em córregos de $3^{\mathrm{a}}$ à $5^{\mathrm{a}}$ ordem preservados e alterados. 
Comentários: T. yura é uma espécie descrita para a Bolívia e o primeiro registro para o Brasil foi em 2010 por Salles et al. (2010b). Esse é o primeiro registro desta espécie para o Estado do Mato Grosso.

Material examinado: PT03: (1) 13.i.2005, PT04: (2) 11.vii.2005, PT04: (2) 19.x.2005, PT11: (1) 25.x.2005, PT12: (1) 12.i.2005, PT19: (1) 19.i.2005, PT19: (1) 19.i.2005, PT19: (2) 21.x.2005, PT19: (3) 18.vii.2005, PT20: (1) 18.vii.2005, PT20: (2) 21.x.2005, PT20: (5) 19.i.2005, PT22: (1) 04.ix.2005.

Tricorythopsis bahiensis Dias, Salles \& Ferreira, 2008

Distribuição: AM, BA, MT e RR.

Aspectos biológicos: Coletado apenas na $1^{\mathrm{a}}$ ordem do Córrego da Mata, associado ao substrato pedra, e na $3^{\mathrm{a}}$ ordem do Córrego Papagaio, ambos da Bacia do Rio Pindaíba.

Comentários: Primeiro registro do gênero e espécie para o Estado do Mato Grosso.

Material examinado: PT09: (2) 09.i.2005; PT09: (1) 04.v.2008; PT15: (1) 08.i.2005.

Tricorythopsis cf. baptistai Dias \& Salles, 2005

Distribuição: MG e MT.

Aspectos biológicos: Foi amostrado apenas um indivíduo desta espécie na $3^{\mathrm{a}}$ ordem do Córrego da Mata.

Comentários: Esta espécie apresentou todas as características morfológicas de acordo com a descrição de Dias \& Salles (2005), porém, o padrão de coloração do abdome e das brânquias se apresentou muito diferente da descrição original, por isso, como se obteve apenas um exemplar desta espécie, consideramos que mais exemplares devem ser examinados a fim de confirmarmos a sua identificação. Esse é o primeiro registro desta espécie para o Mato Grosso.

Material examinado: PT11: (1) 18.i.2008.

Tricorythopsis chiriguano Molineri, 2001

Distribuição: MT.

Aspectos biológicos: Coletada apenas na Bacia do Rio Pindaíba, esta espécie foi amostrada em $2^{\mathrm{a}}, 3^{\mathrm{a}}$ e $4^{\mathrm{a}}$ ordens de córregos considerados conservados.

Comentários: Esse é o primeiro registro da espécie no Brasil, há registros anteriores de T. chiriguano para a Bolívia (Domínguez et al. 2006).

Material examinado: PT03: (1) 12.vii.2005; PT12: (1) 12.i.2005; PT14: (1) 07.i.2005; PT14: (1) 20.xi.2005; PT16: (1) 08.i.2005; PT16: (2) 19.xi.2005; PT18: (1) 20.x.2005; PT19: (1) 18.vii.2005; PT20: (1) 19.i.2005.

\section{Leptophlebiidae}

Estabelecido como família em 1900 por Banks, este grupo compõe um elemento dominante em córregos de pequeno a médio porte, sendo provavelmente a família mais diversa na Região Neotropical, seguido de Baetidae (Savage 1987, Domínguez et al. 2006). Amplamente distribuída, a família alcança seu máximo de diversidade no Hemisfério Sul (Edmunds Junior et al. 1976).

De acordo com Edmunds Junior et al. (1976), as ninfas de Leptophlebiidae são encontradas em fendas embaixo de pedras e troncos em córregos, bem como entre acúmulos de folhas com silte ou embaixo de acúmulos (tapetes) de algas em porções de meandros em córregos, ocupam, também, as margens de lagos onde há movimento da água, ocupando fendas em objetos firmemente ancorados no chão.

No Brasil são descritos 23 gêneros e 53 espécies, sendo que algumas foram descritas para o Mato Grosso recentemente (e.g. Polegatto \& Batista 2007).
Askola sp.

Aspectos biológicos: Esse gênero contribuiu com apenas uma espécie não identificada, amostrados em ambientes de $1^{\mathrm{a}}$ a $4^{\mathrm{a}}$ ordem na Bacia do Rio Pindaíba, e em ambientes de pequeno a médio porte da Bacia do Rio Suiá-Miçú.

Comentários: Esse gênero apresenta apenas uma espécie à qual a ninfa é descrita, Askola froehlichi Peters, 1969, que possui características diferentes das Askola coletadas no presente estudo. Esse é o primeiro registro do gênero para o Estado.

Material examinado: PT02: (2) 18.xi.2005; PT03: (10) 13.i.2005; PT03: (8) 19.x.2005; PT06: (1) 04.xi.2007; PT09: (1) 09.i.2005; PT10: (1) 03.viii.2007; PT10: (1) 09.ii.2005; PT10: (4) 25.x.2005; PT11: (1) 05.v.2008; PT12: (1) 05.xi.2007; PT12: (2) 17.xi.2005; PT13: (2) 16.i.2005; PT13: (5) 20.xi.2005; PT14: (1) 07.i.2005; PT14: (1) 14.vii.2005; PT14: (1) 20.xi.2005; PT15: (3) 19.xi.2005; PT17: (1) 15.i.2005; PT17: (6) 15.i.2005; PT25: (1) 23.v.2008; PT25: (1) 27.ix.2007; PT31: (2) 23.ix.2007; PT32: (1) 20.xii.2007; PT32: (3) 23.ix.2007; PT35: (1) 16.xii.2007.

Farrodes spp.

Aspectos biológicos: Gênero abundante e amplamente distribuído, o qual é composto provavelmente por várias espécies não identificadas, foi amostrado em todos os pontos de coleta da Bacia do Rio Pindaíba, enquanto que no Rio Suiá-Miçú foi presente em córregos e brejos.

Comentários: O gênero Farrodes é bastante diverso, com 12 espécies presentes na America do Sul, porém é praticamente impossível a identificação dos indivíduos em nível específico com base em ninfas (Domínguez et al. 2006).

Material examinado: PT01: (1) 12.vii.2005; PT01: (2) 13.i.2005; PT02: (14) 13.i.2005; PT02: (14) 13.i.2005; PT02: (204) 13.i.2005; PT03: (19) 12.vii.2005; PT03: (21) 19.x.2005; PT03: (31) 13.i.2005; PT04: (13) 08.i.2005; PT04: (14) 19.x.2005; PT04: (5) 19.x.2005; PT04: (9) 11.vii.2005; PT05: (1) 03.viii.2007; PT07: (15) 03.xi.2007; PT07: (2) 03.v.2008; PT07: (2) 04.viii.2007; PT07: (7) 19.i.2008; PT09: (10) 24.x.2005; PT09: (14) 02.viii.2007; PT09: (22) 21.vii.2005; PT09: (30) 09.i.2005; PT09: (31) 02.xi.2007; PT09: (7) 17.i.2008; PT10: (14) 04.v.2008; PT10: (22) 02.xi.2007; PT10: (46) 09.i.2005; PT10: (48) 03.viii.2007; PT10: (66) 09.viii.2005; PT10: (70) 09.viii.2005; PT10: (7) 17.i.2008; PT11: (30) 05.xi.2007; PT11: (36) 25.x.2005; PT11: (38) 01.viii.2007; PT11: (57) 05.v.2008; PT11: (5) 18.i.2008; PT11: (72) 13.vii.2005; PT11: (88) 12.i.2005; PT12: (10) 18.i.2008; PT12: (2) 05.xi.2007; PT12: (40) 17.xi.2005; PT12: (42) 12.i.2005; PT12: (47) 05.v.2008; PT12: (66) 01.viii.2007; PT12: (80) 16.vii.2005; PT13: (35) 14.vii.2005; PT13: (39) 20.xi.2005; PT13: (50) 16.i.2005; PT14: (25) 07.i.2005; PT14: (31) 20.xi.2005; PT14: (66) 14.vii.2005; PT15: (16) 08.i.2005; PT15: (54) 19.xi.2005; PT15: (56) 19.vii.2005; PT16: (15) 19.xi.2005; PT17: (11) 20.x.2005; PT17: (38) 15.i.2005; PT17: (9) 15.vii.2005; PT18: (1) 15.vii.2005; PT18: (3) 15.i.2005; PT18: (5) 20.x.2005; PT19: (1) 21.x.2005; PT19: (21) 18.vii.2005; PT19: (8) 19.i.2005; PT20: (11) 19.i.2005; PT20: (1) 21.x.2005; PT21: (3) 16.vii.2005; PT21: (3) 28.i.2005; PT22: (1) 04.ix.2005; PT25: (1) 19.xii.2007; PT25: (3) 27.ix.2007; PT25: (8) 23.v.2008; PT26: (2) 25.ix.2007; PT32: (10) 23.ix.2007; PT32: (1) 24.v.2008; PT33: (2) 22.ix.2007; PT35: (1) 16.xii.2007.

Fittkaulus cururuensis Savage, 1986

Distribuição: ES, MT e PA.

Aspectos biológicos: Abundante e amplamente distribuída, esta espécie foi amostrada em quase todos os pontos da Bacia do Rio Pindaíba, com exceção da $3^{\mathrm{a}}$ ordem do Córrego Taquaral. Na Bacia do Rio Suiá-Miçú foi presente em sete dos 12 córregos amostrados. 
Comentários: Esta espécie foi registrada para o Mato Grosso por Boldrini et al. (2009).

Material examinado: PT01: (1) 12.vii.2005; PT02: (31) 13.i.2005; PT02: (7) 12.vii.2005; PT03: (10) 19.x.2005; PT03: (2) 12.vii.2005; PT03: (8) 13.i.2005; PT04: (6) 11.vii.2005; PT08: (1) 06.viii.2007; PT08: (2) 20.v.2008; PT10: (1) 09.i.2005; PT10: (3) 02.xi.2007; PT11: (10) 12.i.2005; PT11: (19) 05.xi.2007; PT11: (2) 05.v.2008; PT11: (2) 13.vii.2005; PT11: (3) 01.viii.2007; PT11: (8) 25.x.2005; PT12: (10) 12.i.2005; PT12: (1) 18.i.2008; PT12: (2) 01.viii.2007; PT12: (2) 05.xi.2007; PT12: (3) 16.vii.2005; PT12: (6) 17.xi.2005; PT14: (1) 08.i.2005; PT14: (1) 14.vii.2005; PT14: (1) 19.vii.2005; PT14: (2) 19.xi.2005; PT14: (6) 07.i.2005; PT15: (15) 19.xi.2005; PT15: (2) 08.i.2005; PT15: (6) 19.vii.2005; PT17: (1) 15.vii.2005; PT17: (31) 20.x.2005; PT18: (1) 20.x.2005; PT18: (3) 15.vii.2005; PT20: (3) 21.x.2005; PT21: (2) 26.x.2005; PT21: (4) 28.i.2005; PT22: (1) 15.xi.2005; PT22: (21) 20.i.2005; PT23: (1) 20.i.2005; PT23: (4) 15.xi.2005; PT24: (10) 24.ix.2007; PT24: (11) 25.v.2008; PT24: (1) 16.xii.2007; PT25: (3) 18.xii.2007; PT25: (4) 26.v.2008; PT25: (6) 25.ix.2007; PT29: (1) 27.v.2008; PT30: (11) 26.ix.2007; PT30: (1) 17.xii.2007; PT30: (2) 27.v.2008; PT32: (4) 24.v.2008; PT32: (5) 23.ix.2007; PT33: (1) 20.xii.2007; PT33: (2) 24.v.2008; PT33: (3) 23.ix.2007; РT35: (27) 25.v.2008; РT35: (5) 24.ix.2007; PT35: (8) 16.xii.2007.

\section{Hagenulopsis sp.}

Aspectos biológicos: Composto por apenas uma espécie não identificada, este gênero foi amostrado em córregos de $1^{\mathrm{a}} \mathrm{a} 4^{\mathrm{a}}$ ordem na Bacia do Rio Pindaíba, e apenas em dois pequenos córregos da Bacia do Rio Suiá-Miçú.

Comentários: Esse morfótipo não foi identificado a nível específico, pois a taxonomia é baseada principalmente na morfologia dos adultos, assim como a maioria dos gêneros de Leptophlebiidae. Esse é o primeiro registro do gênero para o estado.

Material examinado: PT02: (1) 13.i.2005; PT02: (3) 12.vii.2005; PT03: (6) 19.x.2005; PT03: (76) 13.i.2005; PT03: (8) 12.vii.2005; PT09: (1) 17.i.2005; PT09: (1) 17.i.2008; PT09: (1) 21.vii.2005; PT09: (2) 02.viii.2007; PT09: (4) 24.x.2005; PT09: (4) 24.x.2005; PT09: (8) 09.i.2005; PT10: (1) 09.i.2005; PT10: (1) 17.i.2008; PT10: (2) 03.viii.2007; PT11: (1) 25.x.2005; PT11: (3) 01.viii.2007; PT11: (4) 12.i.2005; PT11: (4) 13.vii.2005; PT12: (2) 12.i.2005; PT13: (17) 14.vii.2005; PT13: (28) 16.i.2005; PT14: (11) 14.vii.2005; PT14: (1) 07.i.2005; PT15: (3) 08.i.2005; PT15: (3) 19.xi.2005; PT17: (1) 15.i.2005; PT17: (2) 20.x.2005; PT18: (2) 20.x.2005; PT18: (3) 15.vii.2005; PT18: (4) 15.i.2005; PT25: (1) 23.v.2008; PT32: (1) 23.ix.2007.

Hydrosmastodon sallesi Polegatto \& Batista, 2007

Distribuição: MT e RR.

Aspectos biológicos: Foram coletados apenas dois indivíduos na $4^{\mathrm{a}}$ ordem do Córrego da Mata, um córrego largo com mata ciliar bem preservada.

Comentários: Esta espécie já foi registrada para o estado por Polegatto \& Batista (2007).

Material examinado: PT12: (2) 18.i.2008.

Hydrosmilodon gilliesae Thomas \& Péru, 2004

Distribuição: ES e MT.

Aspectos biológicos: Coletados em ambientes de $3^{\mathrm{a}}$ a $6^{\mathrm{a}}$ ordem na Bacia do Rio Pindaíba, principalmente na $3^{\mathrm{a}}$ ordem do Córrego Caveira, um trecho bastante impactado, com ausência quase total da vegetação ribeirinha devido ao uso da terra pela agropecuária. No Rio Suiá-Miçú foram coletados indivíduos em ambientes lóticos e brejosos.
Comentários: Esse é o primeiro registro do gênero e espécie para o Mato Grosso.

Material examinado: PT07: (13) 19.i.2008; PT07: (1) 03.v.2008; PT07: (4) 04.viii.2007; PT07: (6) 03.xi.2007; PT11: (1) 05.v.2008; PT12: (1) 18.i.2008; PT22: (1) 04.ix.2005; PT23: (1) 04.ix.2005; PT24: (1) 24.ix.2007; PT31: (1) 20.xii.2007; PT31: (1) 23.ix.2007; PT32: (1) 24.v.2008; PT32: (8) 23.ix.2007; PT33: (1) 24.v.2008; PT33: (2) 23.ix.2007.

\section{Microphlebia surinamensis Savage \& Peters, 1983}

Distribuição: MT.

Aspectos biológicos: Foram amostrados três indivíduos na $3^{\text {a }}$ ordem do Córrego Caveira (impactado), enquanto que na Bacia do Rio Suiá-Miçú a espécie foi bem distribuída, ocorrendo em seis dos 12 córregos amostrados, todos de águas mais lentas, em áreas alagáveis com presença de muitas macrófitas e buritizais.

Comentários: Anteriormente registrado para o Suriname e Venezuela, esse é o primeiro registro de $M$. surinamensis para o Brasil (Savage \& Peters 1983, Domínguez et al. 2006).

Material examinado: PT07: (3) 03.xi.2007; PT24: (16) 24.ix.2007; PT24: (2) 25.v.2008; PT26: (12) 26.v.2008; PT26: (18) 25.ix.2007; PT26: (2) 18.xii.2007; PT27: (1) 27.ix.2007; PT30: (4) 26.ix.2007; PT30: (5) 27.v.2008; PT34: (2) 26.v.2008; РT35: (2) 25.v.2008

\section{Miroculis spp.}

Aspectos biológicos: Gênero abundante e amplamente distribuído no Mato Grosso, composto provavelmente por mais de uma espécie não identificada. Amostrado em trechos de $1^{\mathrm{a}}$ a $5^{\mathrm{a}}$ ordem na Bacia do Rio Pindaíba, e no Rio Suiá-Miçú em nove dos 12 pontos amostrados, com exceção de três ambientes muito alterados (PT27, PT32 e PT33).

Comentários: De acordo com Domínguez et al. (2006), ninfas e fêmeas não são separáveis em nível subgenérico, sendo a taxonomia baseada principalmente nas características dos olhos dos machos.

Material examinado: PT01: (12) 12.vii.2005; PT01: (53) 13.i.2005; PT02: (175) 12.vii.2005; PT02: (365) 13.i.2005; PT03: (131) 12.vii.2005; PT03: (268) 19.x.2005; PT03: (42) 13.i.2005; PT04: (10) 11.vii.2005; PT04: (33) 19.x.2005; PT04: (6) 08.i.2005; PT05: (1) 03.viii.2007; PT06: (1) 04.xi.2007; PT07: (1) 03.v.2008; PT07: (3) 03.xi.2007; PT08: (1) 03.v.2008; PT08: (1) 03.xi.2007; PT09: (11) 02.viii.2007; PT09: (2) 17.i.2005; PT09: (5) 02.xi.2007; PT10: (10) 02.xi.2007; PT10: (11) 09.viii.2005; PT10: (11) 25.x.2005; PT10: (17) 03.viii.2007; PT10: (1) 09.i.2005; PT11: (110) 05.xi.2007; PT11: (119) 01.viii.2007; PT11: (129) 13.vii.2005; PT11: (17) 12.i.2005; PT11: (1) 05.v.2008; PT11: (1) 18.i.2008; PT11: (45) 25.x.2005; PT12: (11) 05.v.2008; PT12: (17) 17.xi.2005; PT12: (24) 05.xi.2007; PT12: (29) 01.viii.2007; PT12: (40) 16.vii.2005; PT12: (5) 12.i.2005; PT12: (6) 18.i.2008; PT12: (8) 08.i.2005; PT13: (1) 07.i.2005; PT13: (20) 14.vii.2005; PT13: (4) 20.xi.2005; PT14: (17) 19.xi.2005; PT14: (23) 19.xi.2005; PT14: (27) 19.vii.2005; PT14: (4) 08.i.2005; PT14: (4) 08.i.2005; PT14: (95) 19.vii.2005; PT17: (10) 15.vii.2005; PT17: (15) 15.i.2005; PT17: (6) 20.x.2005; PT18: (122) 15.i.2005; PT18: (50) 20.x.2005; PT18: (94) 15.vii.2005; PT19: (111) 21.x.2005; PT19: (5) 19.i.2005; PT19: (74) 21.x.2005; PT20: (20) 19.i.2005; PT20: (84) 18.vii.2005; PT21: (13) 16.vii.2005; PT21: (15) 26.x.2005; PT21: (22) 28.i.2005; PT21: (22) 28.i.2005; PT24: (1) 24.ix.2007; PT25: (1) 27.ix.2007; PT25: (4) 23.v.2008; PT26: (1) 26.v.2008; PT30: (11) 27.v.2008; PT30: (27) 26.ix.2007; PT30: (4) 17.xii.2007; PT31: (18) 23.ix.2007; PT31: (1) 20.xii.2007; PT31: (3) 24.v.2008; РT32: (6) 23.ix.2007; РT33: (3) 20.xii.2007; PT33: (6) 23.ix.2007; РT34: (2) 18.xii.2007; PT34: (2) 25.ix.2007; PT35: (1) 16.xii.2007; PT35: (3) 25.v.2008. 
Paramaka convexa (Spieth, 1943)

Distribuição: MT e PA.

Aspectos biológicos: Coletada em ambientes de $1^{\mathrm{a}}$ a $5^{\mathrm{a}}$ ordem na Bacia do Rio Pindaíba e com apenas um exemplar amostrado em um córrego levemente antropizado na Bacia do Rio Suiá-Miçú.

Comentários: Esse é o primeiro registro do gênero e espécie P. convexa para o Estado do Mato Grosso.

Material examinado: PT03: (1) 12.vii.2005; PT03: (8) 13.i.2005; PT04: (8) 18.1.2005; PT07: (16) 04.viii.2007; PT07: (1) 03.v.2008; PT07: (4) 03.xi.2007; PT07: (6) 19.i.2008; PT11: (2) 13.vii.2005; PT14: (1) 01.i.2005; PT15: (2) 08.i.2005; PT17: (1) 15.i.2005; PT19: (1) 21.x.2005; PT19: (8) 19.i.2005; PT20: (12) 19.i.2005; PT22: (4) 04.ix.2005; PT33: (1) 23.ix.2007.

\section{Simothraulopsis sp.}

Aspectos biológicos: Esse gênero, representado por apenas uma espécie não identificada, foi amostrado em trechos de $1^{\mathrm{a}}$ a $4^{\mathrm{a}}$ ordem na Bacia do Rio Pindaíba, e em ambientes alagáveis e lóticos de pequeno, médio e grande porte (até $73 \mathrm{~m}$ de largura).

Comentários: Essa morfoespécie não pode ser identificada a nível específico, pois a taxonomia é baseada principalmente na morfologia dos adultos, assim como a maioria dos gêneros de Leptophlebiidae. Este é o primeiro registro do gênero para o estado.

Material examinado: PT04: (1) 18.i.2005; PT04: (2) 19.x.2005; PT06: (1) 04.xiii.2007; PT06: (4) 19.i.2008; PT07: (3) 04.viii.2007; PT07: (3) 19.i.2008; PT07: (4) 03.xi.2007; PT07: (9) 03.v.2008; PT08: (3) 03.v.2008; PT09: (1) 21.vii.2005; PT10: (1) 09.viii.2005; PT11: (1) 18.i.2008; PT11: (2) 12.i.2005; PT11: (6) 05.xi.2007; PT11: (6) 25.x.2005; PT12: (1) 12.i.2005; PT12: (2) 17.xi.2005; PT12: (6) 05.v.2008; PT13: (2) 14.vii.2005; PT14: (1) 14.vii.2005; PT15: (1) 19.xi.2005; PT16: (1) 19.xi.2005; PT19: (2) 21.x.2005; PT20: (1) 18.vii.2005; PT20: (6) 21.x.2005; PT24: (3) 25.v.2008; PT24: (6) 24.ix.2007; PT25: (1) 19.xii.2007; PT25: (1) 27.ix.2007; PT25: (4) 23.v.2008; PT26: (17) 26.v.2008; PT26: (18) 25.ix.2007; PT26: (8) 18.xii.2007; PT30: (4) 17.xii.2007; PT30: (6) 27.v.2008; PT30: (7) 26.ix.2007; PT32: (2) 24.v.2008; PT32: (8) 23.ix.2007; PT33: (2) 24.v.2008; PT34: (3) 18.xii.2007; PT34: (3) 26.v.2008; PT34: (4) 25.ix.2007; PT35: (2) 16.xii.2007; PT35: (5) 25.v.2008.

Terpides sooretamae Boldrini \& Salles 2009

Distribuição: ES e MT.

Aspectos biológicos: Foi amostrada com freqüência em trechos de $1^{\text {a }}$ a $5^{\mathrm{a}}$ ordem na Bacia do Rio Pindaíba, e não foi amostrada na Bacia do Rio Suiá-Miçú.

Comentários: Esta espécie foi descrita recentemente e registrada para o Mato Grosso por Boldrini et al. (2009).

Material examinado: PT01: (1) 13.i.2005; PT02: (1) 12.vii.2005; PT02: (2) 12.vii.2005; PT02: (2) 19.x.2005; PT05: (1) 03.viii.2007; PT07: (4) 03.xi.2007; PT08: (1) 20.i.2008; PT09: (2) 02.xi.2007; PT09: (3) 02.viii.2007; PT09: (3) 09.i.2005; PT10: (17) 17.i.2008; PT10: (19) 02.xi.2007; PT10: (19) 09.i.2005; PT10: (25) 03.viii.2007; PT10: (2) 04.v.2008; PT10: (31) 09.viii.2005; PT10: (76) 25.x.2005; PT11: (134) 13.vii.2005; PT11: (149) 25.x.2005; PT11: (155) 05.xi.2007; PT11: (24) 01.viii.2007; PT11: (42) 12.i.2005; PT11: (5) 05.v.2008; PT11: (8) 18.i.2008; PT12: (16) 17.xi.2005; PT12: (40) 12.i.2005; PT12: (41) 05.v.2008; PT12: (41) 05.v.2008; PT12: (44) 16.vii.2005; PT12: (4) 05.xi.2007; PT12: (5) 01.viii.2007; PT12: (5) 18.i.2008; PT13: (25) 14.vii.2005; PT13: (26) 16.i.2005; PT13: (43) 20.xi.2005; PT14: (16) 07.i.2005; PT15: (17) 19.vii.2005; PT15: (1) 08.i.2005; PT15: (4) 19.xi.2005; PT15: (55) 19.vii.2005; PT17: (101) 20.x.2005; PT17: (135) 15.vii.2005; PT17: (154) 15.i.2005; PT18: (27) 15.i.2005; PT18: (7) 20.x.2005; PT18: (8) 15.vii.2005; PT21: (1) 04.ix.2005; PT21: (1) 20.i.2005.

\section{Thraulodes sp.}

Aspectos biológicos: Coletado apenas um indivíduo na Bacia do Rio Pindaíba, na $1^{\text {a }}$ ordem do Córrego Taquaral, e na Bacia do Rio Suiá-Miçú, em apenas dois córregos. Em ambas as bacias, os ambientes em que a espécie foi amostrada são relativamente preservados, de águas lóticas e de pequenas larguras (9 $\mathrm{m}$ de largura).

Comentários: Assim como outros gêneros (Hagenulopsis, Traverella), essa morfoespécie não foi identificada a nível específico, pois sua taxonomia é baseada principalmente na morfologia dos adultos. Esse é o primeiro registro do gênero para o Mato Grosso.

Material examinado: PT17: (1) 15.vii.2005; PT32: (2) 23.ix.2007; PT33: (3) 23.ix.2007.

Tikuna bilineata (Needham \& Murphy, 1924)

Distribuição: PA e MT.

Aspectos biológicos: Coletados no Córrego Taquaral, em $3^{\mathrm{a}}$ e $4^{\mathrm{a}}$ ordens, sendo que apenas um indivíduo foi coletado no Córrego da Mata $2^{\mathrm{a}}$ ordem. Ambos os ambientes apresentam suas matas ciliares relativamente conservadas.

Comentários: Esta espécie foi registrada para o Mato Grosso por Boldrini et al. (2009).

Material examinado: PT18: (1) 14.xi.2005; PT19: (1) 19.i.2005; PT20: (1) 21.x.2005.

\section{Traverella sp.}

Aspectos biológicos: Foi amostrado apenas na Bacia do Rio Pindaíba, nas $3^{\mathrm{a}}$ e $4^{\mathrm{a}}$ ordens, alguns indivíduos associados ao substrato madeira.

Comentários: Esse gênero não foi identificado a nível específico uma vez que a morfologia é baseada principalmente em adultos. Esse é o primeiro registro do gênero para o estado.

Material examinado: PT19: (15) 19.i.2005; PT20: (2) 19.i.2005.

\section{Ulmeritoides sp.1}

Aspectos biológicos: Essa morfoespécie foi abundante e bem distribuída nas bacias dos rios Pindaíba e Suiá-Miçú, ocorrendo em trechos de $1^{\mathrm{a}}$ a $6^{\mathrm{a}}$ ordem da Bacia do Rio Pindaíba, e em dez dos 12 pontos amostrados na Bacia do Rio Suiá-Miçú, não ocorrendo apenas nos PT25 e PT27, ambientes com alto nível de conservação e degradação, respectivamente.

Comentários: Essa morfoespécie é caracterizada por apresentar espinhos posterolaterais nos segmentos abdominais VI-IX, porém não foi identificada a nível específico devido à carência de chaves de identificação e descrição de ninfas desse gênero. A maioria das espécies descritas foi baseada em imagos.

Material examinado: PT01: (1) 13.i.2005; PT01: (7) 12.vii.2005; PT02: (2) 18.i.2005; PT02: (6) 18.xi.2005; PT03: (3) 19.x.2005; PT03: (4) 12.vii.2005; PT04: (1) 11.vii.2005; PT04: (2) 19.x.2005; PT10: (1) 09.i.2005; PT10: (2) 25.x.2005; PT11: (13) 12.i.2005; PT11: (16) 13.vii.2005; PT11: (3) 25.x.2005; PT12: (4) 17.xi.2005; PT12: (7) 16.vii.2005; PT14: (1) 14.vii.2005; PT15: (2) 19.xi.2005; PT16: (11) 19.vii.2005; PT16: (6) 19.xi.2005; PT17: (6) 15.vii.2005; PT18: (17) 15.vii.2005; PT18: (6) 20.x.2005; PT19: (14) 21.x.2005; PT19: (1) 18.vii.2005; PT20: (16) 21.x.2005; PT20: (2) 18.vii.2005; PT21: (1) 26.x.2005; PT21: (2) 28.i.2005; PT22: (1) 04.ix.2005; PT22: (2) 15.xi.2005; PT22: (2) 20.i.2005; PT23: (2) 15.xi.2005; PT24: (3) 24.ix.2007; PT26: (2) 26.v.2008; PT26: (4) 25.ix.2007; PT28: (1) 27.v.2008; PT29: (1) 28.ix.2007; PT30: (11) 27.v.2008; PT30: (4) 17.xii.2007; PT30: (6) 26.ix.2007; PT31: (1) 20.xii.2007; PT31: (1) 23.ix.2007; PT32: (1) 23.ix.2007; РT33: (10) 26.v.2008; PT33: (15) 25.ix.2007; PT33: (3) 18.xii.2007; PT34: (11) 16.xii.2007; PT34: (3) 24.ix.2007; PT34: (3) 25.v.2008. 


\section{Ulmeritoides $\mathrm{sp} .2$}

Aspectos biológicos: Altamente abundante, essa morfoespécie foi presente apenas na Bacia do Rio Pindaíba, em todas as ordens amostradas, com exceção da $5^{\text {a }}$ ordem.

Comentários: Essa morfoespécie foi diferenciada da sp.1 devido aos espinhos posterolaterais do abdômen, que são presentes nos segmentos II-IX. Atualmente, a única espécie de Ulmeritoides que apresenta espinhos nos segmentos II-IX é Ulmeritoides uruguayensis (Traver), 1991, porém, a morfoespécie registrada aqui, não possui a mancha preta na tíbia mediana, característica de $U$. uruguayensis.

Material examinado: PT01: (4) 13.i.2005; PT01: (61) 12.vii.2005; PT02: (19) 13.i.2005; PT02: (2) 12.vii.2005; PT02: (7) 18.xi.2005; PT03: (1) 19.x.2005; PT03: (4) 12.vii.2005; PT03: (4) 13.i.2005; PT04: (4) 11.vii.2005; PT10: (1) 09.i.2005; PT11: (12) 13.vii.2005; PT11: (15) 12.i.2005; PT11: (2) 25.x.2005; PT12: (25) 12.i.2005; PT12: (8) 16.vii.2005; PT15: (2) 19.xi.2005; PT16: (2) 08.i.2005; PT17: (11) 15.vii.2005; PT17: (2) 20.x.2005; PT17: (7) 15.i.2005; PT18: (12) 15.vii.2005; PT18: (34) 15.i.2005; PT18: (3) 20.x.2005; PT19: (1) 18.vii.2005; PT20: (1) 21.x.2005; PT23: (2) 15.xi.2005.

\section{Oligoneuriidae}

Esta família é distribuída na América do Norte e do Sul, Europa, Ásia e África e contêm três subfamílias (Chromarcynae, Colocrurinae e Oligoneurinae), sendo que Colocrurinae esta extinta (Domínguez et al. 2006).

Os indivíduos desta família são encontrados apenas em ambientes lóticos. Os integrantes do gênero Homoeoneuria Eaton, 1881 vivem parcialmente enterrados em áreas com areia, enquanto que Lachlania Hagen, 1868, Oligoneuria Pictet, 1843 e Fittkauneuria Pescados \& Edmunds, 1994 vivem em áreas de forte correnteza, em galhos, raízes ou mesmo em pedras (Salles 2006).

No Brasil são conhecidos seis gêneros e nove espécies de Oligoneuriidae, sendo que até o presente momento apenas uma espécie é registrada para o estado (Oligoneuria amazonica Demoulin, 1955) (Salles 2010).

Lachlania sp.

Aspectos biológicos: Coletados apenas na Bacia do Rio Pindaíba, em trechos de $1^{\text {a }}$ a $4^{\mathrm{a}}$ ordem. Indivíduos desta família não foram coletados na Bacia do Rio Suiá-Miçú.

Material examinado: PT03: (14) 13.i.2005; PT09: (1) 09.i.2005; PT10: (8) 09.i.2005; PT11: (8) 12.i.2005; PT12: (2) 12.i.2005; PT14: (1) 07.i.2005; PT15: (10) 08.i.2005.

Oligoneuria amazonica (Demoulin, 1955)/ SINÔNIMOS=Oligoneurioides amazonicus Demoulin, 1955

Distribuição: AM, MA, MT, PA, RO e RR.

Aspectos biológicos: Coletados em trechos de $3^{\mathrm{a}}, 4^{\mathrm{a}}$ e $5^{\mathrm{a}}$ ordens na Bacia do Rio Pindaíba.

Comentários: Esta espécie foi registrada para o Mato Grosso por Salles et al. (2007).

Material examinado: PT03: (3) 13.i.2005; PT19: (1) 19.i.2005; PT20: (10) 19.i.2005; PT21: (1) 28.i.2005.

\section{Polymitarcyidae}

De acordo com Domínguez et al. (2006) essa família é amplamente distribuída, sendo composta por três subfamílias: Polymitarcyinae, Asthenopodinae e Campsurinae. Porém, mesmo com 42 espécies descritas em apenas um gênero (Campsurus Eaton, 1868), essa família é a menos conhecida. Trabalhos recentes a respeito do grupo são praticamente inexistentes, sendo a validade de suas espécies muitas vezes questionável (Salles et al. 2004a, Salles 2006).
As descrições de muitas espécies são baseadas principalmente nas fêmeas adultas, o que dificulta a identificação desses organismos em sua fase ninfal ou mesmo imaginal (Domínguez et al. 2006). As ninfas dessa família possuem adaptações morfológicas para escavar e fazem túneis em forma de U, no fundo de habitats lênticos (Edmunds Junior et al. 1976, Domínguez et al. 2006).

No Brasil são descritos quatro gêneros (Tortopus Needham \& Murphy, 1924, Tortopsis Molineri, 2010, Campsurus e Asthenopus Eaton, 1871), todos foram encontrados no Mato Grosso.

Asthenopus curtus (Hagen, 1861)

Distribuição: AM, MT e PA.

Aspectos biológicos: Coletada apenas na Bacia do Rio SuiáMiçú, esta espécie foi característica de ambientes brejosos com acúmulo de matéria orgânica e silte e também foi presente em trechos represados do Rio Betis.

Comentários: Esse é o primeiro registro do gênero e espécie para o Estado do Mato Grosso.

Material examinado: PT06: (1) 04.xi.2007; PT06: (2) 04.viii.2007; PT06: (8) 19.i.2008; PT24: (17) 25.v.2008; PT26: (17) 26.v.2008; PT26: (1) 18.xii.2007; PT29: (2) 17.xii.2007; PT30: (22) 27.v.2008; PT30: (2) 17.xii.2007; РT30: (6) 26.ix.2007.

\section{Campsurus spp.}

Aspectos ecológicos: Esse gênero foi o mais diverso entre os Ephemeroptera, encontradas na Bacia do Rio Pindaíba em ambientes de $1^{\mathrm{a}}$ a $5^{\mathrm{a}}$ ordem e na Bacia do Rio Suiá-Miçu, em oito dos 12 pontos amostrados, de águas lóticas e ambientes brejosos.

Comentários: A identificação de Campsurus não foi possível devido aos poucos estudos com a família e descrições de espécies questionáveis. Ao ser morfotipado, baseado em diferenças nas projeções mandibulares, os representantes de Campsurus contribuíram com dez morfoespécies. Esse é o primeiro registro do gênero para o Mato Grosso.

Material examinado: PT01: (1) 12.vii.2005; PT02: (4) 13.i.2005; РT03: (21) 12.vii.2005; РT03: (1) 12.vii.2005; РT06: (9) 06.v.2008; PT06: (13) 04.viii.2007; PT06: (4) 04.xi.2007; PT07: (3) 19.i.2008; PT07: (10) 03.v.2008; PT07: (1) 04.viii.2007; PT07: (6) 03.xi.2007; PT08: (5) 03.v.2008; PT08: (1) 03.xi.2007; PT09: (1) 17.i.2008; PT34: (24) 18.xii.2007; PT34: (4) 25.ix.2007; PT35: (6) 25.v.2008; PT35: (4) 24.ix.2007; PT35: (3) 16.ix.2007; PT11: (73) 18.i.2008; PT11: (50) 05.v.2008; PT11: (11) 01.viii.2007; PT11: (2) 05.xi.2007; PT11: (33) 12.i.2005; PT11: (2) 13.vii.2005; PT12: (28) 18.i.2008; PT12: (1) 05.v.2008; PT12: (10) 01.viii.2007; PT12: (2) 05.xi.2007; PT12: (12) 12.i.2005; PT26: (1) 25.ix.2007; PT19: (1) 21.x.2005; PT20: (1) 21.x.2005; PT04: (1) 19.x.2005; PT33: (5) 17.xii.2007; PT33: (1) 27.v.2008; РT30: (18) 27.v.2008; PT30: (7) 26.ix.2007; PT30: (3) 17.xii.2007; РT31: (3) 24.v.2008; PT31: (1) 24.v.2008; PT31: (1) 20.xii.2007; PT22: (3) 15.xi.2005; PT22: (2) 04.ix.2005; PT32: (13) 24.v.2008; PT32: (8) 23.ix.2007; PT33: (8) 24.v.2008; PT33: (2) 23.ix.2007; PT33: (6) 20.xii.2007.

\section{Tortopsis sp.}

Distribuição: RJ.

Aspectos biológicos: Foi coletado apenas um indivíduo na $3^{\mathrm{a}}$ ordem do Córrego Cachoeirinha, ambiente alterado.

Comentários: Este gênero foi recentemente desmembrado de Tortopus por Molineri (2010), porém, o único indivíduo amostrado se encontra em um estádio precoce de seu desenvolvimento, o que não permitiu a identificação do mesmo. Esse é o primeiro registro do gênero para o Estado de Mato Grosso.

Material examinado: PT03: (1) 12.vii.2005. 


\section{Conclusão}

Foram identificadas 42 espécies nominais de Ephemeroptera, pertencentes a sete famílias e 28 gêneros. Além dessas espécies, 13 gêneros de seis famílias foram morfoespeciados em 25 táxons: uma morfoespécie de Baetodes, três de Callibaetis, uma de Campylocia, uma de Leptohyphes, uma de Askola, uma de Hagenulopsis, uma de Thraulodes, uma de Traverella, uma de Simothraulopsis, duas de Ulmeritoides, uma de Lachlania, uma de Tortopsis e dez de Campsurus. Alguns problemas relacionados à identificação de ninfas, como falta de chaves de identificação ou semelhança geral entre diferentes espécies de um mesmo gênero, inviabilizaram maiores informações quanto ao número de espécies dos seguintes gêneros: Farrodes, Miroculis e Traverhyphes. Sendo assim, 67 espécies/ morfoespécies foram identificadas em 41 gêneros e oito famílias na região leste do Mato Grosso.

Apesar do grande avanço na taxonomia de Ephemeroptera nos últimos anos, ainda existe uma lacuna na identificação dos indivíduos em sua fase ninfal. É visível a necessidade de novos trabalhos de cunho taxonômico na fase imatura desses organismos, pois são abundantes, de grande importância nas cadeias tróficas nos ecossistemas aquáticos, além de serem utilizados em programas de biomonitoramento de qualidade de água. Uma vez que várias espécies foram descritas tendo como principal caráter a genitália masculina, a criação das ninfas e coleta de adultos provavelmente acarretariam na ampliação de espécies e informações sobre a biologia e diversidade de Ephemeroptera no Mato Grosso.

No presente trabalho, quatro espécies (Cloeodes redactus, Waltzoyphius roberti, Tricorytopsis chiriguano e Microphlebia surinamenses) são registradas pela primeira vez para o Brasil.

Já as famílias Euthyplociidae, Polymitarcyidae e Coryphoridae ampliam a sua distribuição para o Estado do Mato Grosso, assim como os 19 gêneros: Amanahyphes, Askola, Asthenopu,s Baetodes, Campsurus, Campylocia, Coryphorus, Cryptonympha, Guajirolus, Hagenulopsis, Hydrosmilodon, Microphlebia, Paramaka, Simothraulopsis, Thraulodes, Tortopsis, Traverella, Traverhyphes, Tricorythopsis, e 18 espécies: Amanahyphes saguassu, Asthenopus curtus, Caenis cuniana, Caenis fittkaui, Caenis pflugfelderi, Coryphorus aquilus, Cryptonympha copiosa, Guajirolus rondoni, Hydrosmilodon gilliesae, Paramaka convexa, Tricorythodes barbus, Tricorythodes hiemalis, Tricorythodes mirca, Tricorythodes rondoniensis, Tricorythodes santarita, Tricorythodes yura, Tricorythopsis bahiensis e Tricorythopsis cf. baptistai.

Seguindo a tendência dos padrões de diversidade de Ephemeroptera no Brasil observada por Salles et al. (2004b, 2010a) e Francischetti (2007), a família Baetidae foi a mais rica (19 espécies), seguida de Leptophlebiidae (15) e Leptohyphidae (13). Comparando os dados obtidos com os mesmos trabalhos citados acima, a diversidade de Ephemeroptera no Mato Grosso está na média entre a diversidade do Parque Estadual do Rio Doce, Minas Gerais (50 espécies amostradas) e do Espírito Santo (76 espécies). A grande dimensão do território matogrossense $(903.357 \mathrm{~km})$ e a diversidade fitofissiológica do estado (Pantanal, Cerrado, Floresta Amazônica e áreas de transição), que se encontra sobre forte pressão da agricultura e pecuária (principalmente áreas de Cerrado), ressaltam a importância de estudos nas demais localidades do estado para o conhecimento da efêmeropterofauna local. Sendo este levantamento realizado apenas para duas bacias do leste do Estado do Mato Grosso, espera-se que a diversidade de Ephemeroptera seja muito maior, além de provavelmente abrigar várias novas espécies para a Ciência.

\section{Agradecimentos}

Gostaríamos de agradecer ao Herson Lima, Lorivaldo Castro e a equipe do Laboratório de Entomologia da Universidade do Estado do Mato Grosso, Campus de Nova Xavantina, pela ajuda na coleta. Mariana Pavan, Hilton Marcelo Lima Souza e Leandro Brasil pelo auxílio na identificação do material e a Jeane Nascimento pela leitura. Aos proprietários rurais que permitiram e apoiaram a iniciativa deste trabalho. Ao CNPq, Proc. no 520268/2005-9, PROBIO /MMA/, Proc. $n^{\circ}$ 680020/02-0, FAPEMAT, Proc. no 098/2004 e 0907/2006 e PROCAD/CAPES n ${ }^{\circ}$ 109/2007, pelo fomento aos projetos.

\section{Referências Bibliográficas}

BOLDRINI, R. \& SALLES, F.F. 2009. A new species of two-tailed Camelobatidius (Insecta: Ephemeroptera: Baetidae) from the State Espírito Santo. Rev. Mus. Biol. Mello Leitão 25:5-12.

BOLDRINI, R., SALLES, F.F. \& CABETTE, H.R.S. 2009. Contribution to the taxonomy of the Terpides lineage (Ephemeroptera: Leptophlebiidae). Ann. Limnol.-Int. J. Lim. 45:219-229. http://dx.doi.org/10.1051/limn/2009029

BRASIL. 1981. Goiás:geologia, geomorfologia, pedologia, vegetação, uso potencial da terra. Ministério das Minas e Energia, Divisão de Publicação. Projeto RADAMBRASIL, Folha SD 22.

BRITTAIN, J.E. 1982. Biology of Mayflies.Annu. Rev. Entomol. 27:119-147. http://dx.doi.org/10.1146/annurev.en.27.010182.001003

BUSS, D.F. \& SALLES, F.F. 2007.Using Baetidae species as biological indicators of environmental degradation in a Brazilian river basin. Environ. Monit. Assess. 130:365-372. PMid:17106778. http://dx.doi. org/10.1007/s10661-006-9403-6

CRUZ, P.V., SALLES, F.F. \& HAMADA, N. 2009. Two new species of Callibaetis Eaton (Ephemeroptera: Baetidae) from Southeastern Brazil. Zootaxa 2261:23-38.

DA-SILVA, E.R., NESSIMIAN, J.L. \& COELHO, L.B.N. 2010. Leptophlebiidae ocorrentes no Estado do Rio de Janeiro, Brasil: habitats, meso-hábitats e hábitos das ninfas (Insecta: Ephemeroptera). Biota Neotrop. 10(4): http://www.biotaneotropica.org.br/v10n4/en/ abstract?article+bn01410042010 (último acesso em 23/03/2011).

DIAS, L.G. \& SALLES, F.F. 2005. Three new species of Tricorythopsis (Ephemeroptera: Leptohyphidae) from southeastern Brazil. Aquat. Insects 27(4):235-241. http://dx.doi.org/10.1080/01650420500336657

DIAS, L.G., CABETTE H, S.R. \& DE SOUSA, D.P. 2009a. A new species of Tricorythodes Ulmer, 1920 (Ephemeroptera: Leptohyphidae) and first record of Tricorythodes quizeri Molineri, 2002 from Brazil. Aquat. Insects 31(1):95-99. http://dx.doi.org/10.1080/01650420802648858

DIAS, L.G., CRUZ, P.V. \& FERREIRA, P.S.F. 2009b. A new species of Tricorythodes Ulmer (Ephemeroptera: Leptohyphidae) from Northern Brazil. Ann. Limnol. - Int. J. Lim. 45(2):127-129. http://dx.doi. org/10.1051/limn/2009009

DIAS, L.G., SALLES, F.F. \& FERREIRA, P.S.F. 2008. New species of Tricorythopsis Traver (Ephemeroptera: Leptohyphidae) from northern Brazil. Stud. Neotrop. Fauna E. 43(3):237-241. http://dx.doi. org/10.1080/01650520701553826

DIAS, L.G., MOLINERI, C. \& FERREIRA, P.S.F. 2007a. Ephemerelloidea (Insecta: Ephemeroptera) do Brasil. Pap. Avul. Zool. 47(19):213-244.

DIAS, L.G., SALLES, F.F., POLEGATTO, C.M., MARIANO, R.S. \& FROEHLICH, C.G. 2007b. Novos registros de Ephemerelloidea (Insecta: Ephemeroptera) para o estado de São Paulo. Biota Neotrop. 7(3): http:// www.biotaneotropica.org.br/v7n3/pt/abstract?article+bn00307032007 (último acesso em 15/08/2010).

DOMÍNGUEZ, E., MOLINERI, C., PESCADOR, M.L., HUBBARD, M.D. \& NIETO, C. 2006. Ephemeroptera of South America. In Aquatic Biodiversity of Latin America (J. Adis, J.R. Arias, G. Rueda-Delgado \& K.M. Wantzen, eds.). Pensoft, Moscow-Sofia, v.2, p.1-646.

EDMUNDS JUNIOR, G.E., JENSEN, S.L. \& BERNER, L. 1976. The Mayflies of North and Central America. University of Minnesota, Minnesota Press, Minneapolis.

FRANCISCHETTI, C.N. 2007. Ephemeroptera (Insecta) do Parque Estadual do Rio Doce, Minas Gerais, Brasil: biodiversidade e distribuição espacial. Tese de doutorado, Universidade Federal de Viçosa, Viçosa. 
LIMA, L.R.C., SALLES, F.F., PINHEIRO, U.S. \& MIRANDA, E.Q. 2010 Espécies de Baetidae (Ephemeroptera) do sul da Bahia, com descrição de uma nova espécie de Paracloeodes Day. Neotrop. Entomol. 39(5):725-731 PMid:21120380. http://dx.doi.org/10.1590/S1519-566X2010000500009

LUGO-ORTIZ, C.R. \& McCAFFERTY, W.P. 1996. Aturbina georgei gen. and sp. n.: A small minnow mayfly (Ephemeroptera, Baetidae) without turbinate eyes. Aquat. Insects. 18:175-183. http://dx.doi. org/10.1080/01650429609361619

MALZACHER, P. 1998. Remarks on the genus Brasilocaenis (Ephemeroptera: Caenidae), with the description of a new species: Brasilocaenis mendesi. Stuttgarter Beitr. Naturk., Ser. A (Biologie) 580:1-6.

McCAFFERTY, W.P. \& LUGO-ORTIZ, C.R. 1995.Cloeodeshydation, n. sp. (Ephemeroptera: Baetidae): an extraordinary, drought tolerant mayfly from Brazil. Ent. News 106(1):29-35.

McCAFFERTY, W.P., LUGO-ORTIZ, C.R., PROVONSHA, A.V. \& WANG, T.Q. 1997.Los Efemerópteros de México: I. Classificatión superior, diagnoses de famílias e composición. Dugesiana 4(2):1-29.

MELO, A.S. 2008. O que ganhamos 'confundindo' riqueza de espécies e equabilidade em um índice de diversidade? Biota Neotrop. 8(3): http://www.biotaneotropica.org.br/v8n3/en/abstract?point-ofview+bn00108032008 (último acessoem 06/08/2010).

MOLINERI, C. \& MALZACHER, P. 2007. South American Caenis Stephens (Ephemeroptera, Caenidae), new species and stage descriptions. Zootaxa 1660:1-31.

MOLINERI, C. 2010. A cladistic revision of Tortopus Needham \& Murphy with description of the new genus Tortopsis (Ephemeroptera: Polymitarcyidae). Zootaxa. 2481:1-36.

NOLTE, U., OLIVEIRA, M.J. \& STUR, E. 1997. Seasonal, dischargedriven patterns of mayfly assemblages in an intermittent Neotropical stream. Freshwater Biol. 37:333-343. http://dx.doi.org/10.1046/j.13652427.1997.00163.x

PEREIRA, S.M. \& DA-SILVA, E.R. 1990. Nova espécie de Campylocia Needham \& Murphy, 1924 com notas biológicas (Ephemeroptera, Euthyplociidae). Pub. Avul. Mus. Nac. 336:1-12.

PETERS, W.L. 1981. Coryphorusaquilus, a New Genus and Species of Tricorythidae from the Amazon Basin (Ephemoptera). Aquat Insects 3(4):209-217. http://dx.doi.org/10.1080/01650428109361064

POLEGATTO, C.M. \& BATISTA, J.D. 2007. Hydro mastodon sallesi, new genus and new species of Atalophlebiinae (Insecta: Ephemeroptera Leptophlebiidae) from West and North of Brazil, and notes on systematics of Hermanella group. Zootaxa 1619:53-60.

RATTER, J.A., ASKEW, G.P., MONTGOMERY, R.F. \& GIFFORD, D.R. 1978 Observations on the vegetation of northeastern MatoGrosso II.Forest and Soils of the Rio Suiá-Miçu area.Proc. R. Soc. Lond. 293(B):191-208

RATTER, J.A., RIBEIRO, J.F. \& BRIDGEWATER, S. 1997. The Brazilian Cerrado vegetation and threats to its biodiversity. Ann. Bot. 80:223-230. http://dx.doi.org/10.1006/anbo.1997.0469

RIVA, A.L.M., FONSECA, L.F.L. \& HASENCLEVER, L. 2007. Instrumentos econômicos e financeiros para a conservação ambiental no Brasil: Uma análise do estado da arte no Brasil e no Mato Grosso. Instituto Socioambiental-ISA, São Paulo.

ROSSETE, A.N. 2005. Componente: meio físico e uso atual da terra. In Uso de indicadores ambientais na gestão de recursos hídricos na Bacia Hidrográfica do Rio Pindaíba-MT (H.S.R. Cabette, ed.). CNPq, Nova Xavantina, p. 1-21. Relatório Técnico.

SALLES, F.F. 2006. A Ordem Ephemeroptera no Brasil (Insecta): Taxonomia e diversidade. Tese de doutorado, Universidade Federal de Viçosa,Viçosa.

SALLES, F.F. 2010. Lista das espécies de Ephemeroptera registradas para o Brasil. http://ephemeroptera.br.googlepages.com/home (último acesso em 12/08/2010).

SALLES, F.F. \& BATISTA, J.D. 2004. The presence of Varipes Lugo-Ortiz $\&$ McCafferty (Ephemeroptera: Baetidae) in Brazil, with the description of a new species. Zootaxa 456:1-6.
SALLES, F.F. \& BOLDRINI, R. 2008. Male imago description of Americabaetis longetron Lugo-Ortiz \& McCafferty (Ephemeroptera: Baetidae), and first key to adults of the genus. Neotrop. Entomol. 37(5):564-566. PMid:19061042. http://dx.doi.org/10.1590/S1519-566X2008000500010

SALLES, F.F. \& LUGO-ORTIZ, C.R. 2002. A distinctive new species of Apobaetis (Ephemeroptera: Baetidae) from Mato Grosso and Minas Gerais, Brazil. Zootaxa 35:1-6.

SALLES, F.F. \& NASCIMENTO, J.M.C. 2009. The Genus Rivudiva Lugo-Ortiz and McCafferty (Ephemeroptera: Baetidae): First generic description of adults, new combinations, and notes on the nymphs. Ann. Limnol. Int. J. Lim. 45:231-235. http://dx.doi.org/10.1051/limn/2009023

SALLES, F.F. \& NIETO, C. 2008. Los estados adultos de Nanomisy Spiritiops (Ephemeroptera: Baetidae) from Brazil. Rev. Soc. Entomol. Argent. 67(1-2):35-39.

SALLES, F.F. \& POLEGATTO, C.M. 2008. Two new species of Baetodes Needham \& Murphy (Ephemeroptera: Baetidae) from Brazil. Zootaxa 1851:43-50.

SALLES, F.F. \& SERRÃO, J.E. 2005. The nymphs of the genus Camelobaetidius Demoulin (Ephemeroptera :Baetidae) in Brazil: new species, new records, and key for the identification of the species. Ann. Limnol. Int. J. Lim.41:267-279. http://dx.doi.org/10.1051/limn/2005014

SALLES, F.F., BATISTA J.D. \& CABETTE, H.S.R. 2004a. Baetidae (Insecta: Ephemeroptera) de Nova Xavantina, Mato Grosso, Brasil: Novos registros e descrição de uma nova espécie de Cloeodes Traver, Biota Neotropica 4(2): http://www.biotaneotropica.org.br/v4n2/pt/ abstract?article+BN02404022004 (último acesso em 10/08/2010).

SALLES, F.F., BOLDRINI, R., SHIMANO, Y. \& CABETTE. H.S.R. 2010a. Review of the genus Aturbina Lugo-Ortiz \& McCarfferty (Ephemeroptera: Baetidae). Ann. Limnol. 47(1): 21-44. http://dx.doi.org/10.1051/ limn/2010100

SALLES, F.F., DA-SILVA, E.R., HUBBARD, M.D. \& SERRÃO, J.E. 2004 b. As espécies de Ephemeroptera (Insecta) registradas para o Brasil. Biota Neotrop. 4(2): http://www.biotaneotropica.org.br/v4n2/pt/abstract?inve ntory+BN04004022004 (último acesso em 13/08/2010).

SALLES, F.F., FRANCISCHETTI, C.N. \& SOARES, E.D.G. 2009. The presence of Homoeoneurias.s. (Ephemeroptera: Oligoneuriidae) in South America with the description of a new species. Zootaxa 2146:53-60.

SALLES, F.F., NASCIMENTO, J., MASSARIOL, F., ANGELI, K., BARCELOS-SILVA, P., RÚDIO, J. \& BOLDRINI, R. 2010b. First survey of mayflies (Ephemeroptera, Insecta) from Espírito Santo State, Southeastern Brazil. Biota Neotrop. 10(1): http://www.biotaneotropica. org.br/v10n1/en/abstract?inventory+bn02610012010 (último acesso em 09/08/2010).

SALLES, F.F., RAIMUNDI, E.A., BOLDRINI, R. \& SOUZA-FRANCO, G.M. 2010c. The genus Americabaetis Kluge (Ephemeroptera: Baetidae) in Brazil: new species, stage description, and key to nymphs. Zootaxa 2560:16-28.

SALLES, F.F., BAPTISTA, M.S., DA-SILVA, E.R., HAMADA, N. \& SERRÃO, J.E. 2007. Redescription of the adults and description of the larvae and eggs of Oligoneurioides amazonicus Demoulin (Ephemeroptera: Oligoneuriidae). Aquat. Insects 29(2):139-149. http:// dx.doi.org/10.1080/01650420701272305

SAVAGE, H.M \& PETERS, W.L. 1983. Systematics of Miroculis and related genera from northern South America (Ephemeroptera: Leptophlebiidae). T. Am. Entomol. Soc. 108:491-600.

SAVAGE, H.M. 1987. Biogeographic Classification of the Neotropical Leptophlebiidae (Ephemeroptera) based upon Geological Centers of Ancestral Origin and Ecology. Stud. Neotrop. Fauna E. 22(4):199-222. http://dx.doi.org/10.1080/01650528709360734

SCHWARTZMAN, S. \& ZIMMERMAN, B. 2005. Conservation alliances with indigenous peoples of the Amazon. Conserv. Biol. 19(3):721-727. http://dx.doi.org/10.1111/j.1523-1739.2005.00695.x

VIANELLO, R.L. \& ALVES, A.R. 2000. Meteorologia básica e aplicações. Universidade Federal de Viçosa, Viçosa. 
